\title{
Lithium Binaphtholate-Catalyzed Michael Reaction of Malonates with Maleates and Its Application to the Enantioselective Synthesis of Tricarboxylic Acid Derivatives
}

\author{
Midori Sakamoto, Tetsuya Kaneko, Yuya Orito, Yasushi Shimoda,* and Makoto Nakajima* \\ Graduate School of Pharmaceutical Sciences, Kumamoto University; 5-1 Oe-honmachi, Chuo-ku, Kumamoto 862- \\ 0973, Japan.
}

Received December 17, 2018; accepted January 11, 2019

\begin{abstract}
The Michael reaction of malonates with maleates afforded the corresponding adducts in high yields with high enantioselectivities (up to $\mathbf{9 8 \%}$ enantiomeric excess (ee)) by using dilithium $3,3^{\prime}$-dichlorobinaphtholate as a catalyst. The obtained Michael adducts could be converted to optically active tricarboxylic acid (TCA) derivatives via the Krapcho reaction.
\end{abstract} tivity

Key words Michael reaction; lithium binaphtholate; tricarboxylic acid ester; Krapcho reaction; stereoselec-

\section{Introduction}

The Michael reaction of various nucleophiles with acceptors is one of the most versatile tools for the carbon-carbon bond formation reaction. ${ }^{1-7)}$ Enantioselective Michael reaction has emerged as a key step in the construction of complex natural products. Therefore, an elegant and economical Michael reactions have still attracted considerable attentions. To date, numerous catalytic enantioselective Michael reactions have been demonstrated. Generally, enones, ${ }^{8-20)}$ maleimides $^{21,22)}$ and others have been used as Michael acceptors. In contrast, maleates have been used as acceptors to a lesser extent as acceptors. The Michael reaction of malonates to maleates is a typical example of this conjugate addition, which has been previously described even in an early volume of the famous 'Organic Syntheses. ${ }^{23)}$ However, to the best of our knowledge, no reports are available on the enantioselective Michael reactions of malonates to maleates. Our group has reported enantioselective reactions catalyzed by metal binaphtholates. ${ }^{24-33)}$ Metal binaphtholates, ${ }^{34-45)}$ which are easily prepared from 2,2'-binaphthols (BINOLs) and metal bases, such as ${ }^{n} \mathrm{BuLi}$ or Grignard reagents, act as Brønsted bases to promote asymmetric reactions. Recently, we also reported the first example of the enantioselective Michael reaction of malonates to maleates, ${ }^{46)}$ affording tetracarboxylic acid esters as Michael adducts in high yields with high enantioselectivities. Herein, we describe the details of this reaction, including the extension of substrates and the mechanism of lithium binaphtholate-catalyzed Michael reaction of maleates as acceptors. Furthermore, we demonstrate the transformation of obtained product to the tricarboxylic acid (TCA) derivatives, which are often found in biologically active compounds, through decarboxylation. ${ }^{47,48)}$

The initial experiment was started with the addition of dibenzyl malonate (1a) to diethyl maleate (2a) in diethyl ether using lithium binaphtholate catalyst (4a), which was prepared from $(R)-3,3^{\prime}-\mathrm{Cl}_{2}-\mathrm{BINOL}$ and ${ }^{n} \mathrm{BuLi}$. The reaction proceeded smoothly, ${ }^{49)}$ resulting in the corresponding product 3aa in high yield with moderate selectivity within $0.5 \mathrm{~h}$ (Table 1, entry 1). Enantioselectivity decreased using toluene as a solvent, probably owing to the lack of coordination of lithium atom in the solvent (entry 2). Changing the solvent to tert-butyl methyl ether (TBME) and lowering catalyst concentration $(0.01 \mathrm{mmol} / \mathrm{mL})$ dramatically improved the enantioselectivity for 3aa to $90 \%$ enantiomeric excess (ee) (entry 5). Neither lowering the catalyst concentration below $0.01 \mathrm{mmol} / \mathrm{mL}$ nor lowering the temperature $\left(0^{\circ} \mathrm{C}\right)$ increased the enantioselectivity of 3aa (entries 6 and 7). When cyclopentyl methyl ether (CPME) was used as an ether solvent, the same effect as TBME was obtained (entry 8). In the case of 1,2-dimethoxyethane (DME) (entry 9), the Michael reaction did not proceed at all owing to its high chelation ability.

Next, we investigated the effect of the substitution on the 3,3'-position of binaphthols in more detail. The catalyst 4b, prepared from $(R)-3,3^{\prime}-\mathrm{Br}_{2}-\mathrm{BINOL}$ and ${ }^{n} \mathrm{BuLi}$, afforded the Michael adduct in high yield with high enantioselectivity (entry 10), whereas unsubstituted $(R)$-BINOL-Li 2 (4c), $(R)-3,3^{\prime}-\mathrm{Me}_{2}-\mathrm{BINOL}-\mathrm{Li}_{2}$ (4d), and $(R)-3,3^{\prime}-\mathrm{Ph}_{2}-\mathrm{BINOL}-\mathrm{Li}_{2}(\mathbf{4 e})$ were ineffective in this Michael reaction (entries 11-13). From these results, it is suggested that the electron deficiency might increase catalytic activity.

Next, the Michael reactions between various malonates and maleates were carried out under optimized reaction conditions (Table 2). As for the Michael acceptors, reduced stereoselectivities were obtained with dimethyl maleate $(\mathbf{2 b})$ or dibenzyl maleate (2c), or substituted dibenzyl maleates (2d and $\mathbf{2 e}$ ) were found to be decreased (entries 2-4). These results indicate that the electronic and steric effects affect for the reaction rate of this reaction. Di- $n$-propyl maleate (2f) reacted with malonate 1a to give the corresponding Michael adduct 3af, albeit in low enantioselectivity, but diallyl maleate (2g) gave a result similar to that of diethyl maleate (2a). Among the other Michael donors, no alkyl malonate performed better than dibenzyl malonate (1a) (entries 9-11). As the general Michael acceptors, the enantioselectivities of $\mathrm{N}$-phenylmaleimide (2h) and chalcone (2i) were decreased (entries 12, 13). Interestingly, fumarate $\mathbf{2} \mathbf{j}$, the trans-isomer of maleate $\mathbf{2 a}$, gave an almost racemic product 3 aa (entry 14), suggesting that the cis geometry of the $\mathrm{C}-\mathrm{C}$ double bond of $\mathbf{2 a}$ allows both carbonyl groups to coordinate with lithium metals. A ketone with a cis 
Table 1. Optimization of Enantioselective Michael Reaction of Malonate 1a with Maleate 2a
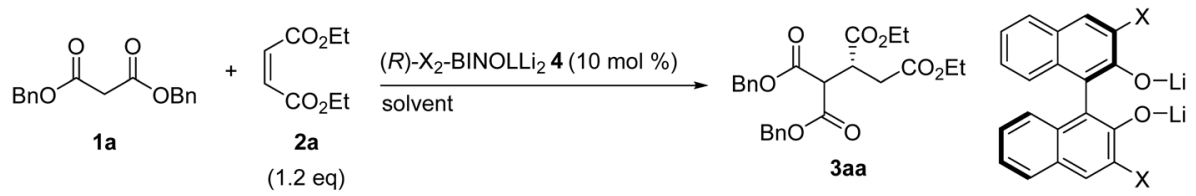

4a: $\mathrm{X}=\mathrm{Cl}$

4b: $X=\mathrm{Br}$

4c: $X=H$

4d: $X=M e$

4e: $\mathrm{X}=\mathrm{Ph}$

$(R)-\mathrm{X}_{2}-\mathrm{BINOLLi}_{2} 4$

\begin{tabular}{|c|c|c|c|c|c|c|}
\hline Entry & Solvent & $c(\mathrm{~mol} / \mathrm{L})(\%)^{a)}$ & Cat.: $\mathrm{X}$ & Conditions & Yield $(\%)^{b)}$ & ee $(\%)^{c, d)}$ \\
\hline 1 & $\mathrm{Et}_{2} \mathrm{O}$ & 0.1 & 4a: $\mathrm{Cl}$ & r.t., $0.5 \mathrm{~h}$ & 93 & $45(R)$ \\
\hline 2 & Toluene & 0.1 & 4a: $\mathrm{Cl}$ & r.t., $0.5 \mathrm{~h}$ & 92 & $20(R)$ \\
\hline 3 & THF & 0.1 & 4a: $\mathrm{Cl}$ & r.t., $4 \mathrm{~h}$ & 85 & $57(R)$ \\
\hline 4 & TBME & 0.1 & 4a: $\mathrm{Cl}$ & r.t., $0.5 \mathrm{~h}$ & 94 & $58(R)$ \\
\hline 5 & TBME & 0.01 & 4a: $\mathrm{Cl}$ & r.t., $1 \mathrm{~h}$ & 94 & $90(R)$ \\
\hline 6 & TBME & 0.003 & 4a: $\mathrm{Cl}$ & r.t., $1 \mathrm{~h}$ & 92 & $90(R)$ \\
\hline 7 & TBME & 0.1 & 4a: $\mathrm{Cl}$ & $0^{\circ} \mathrm{C}, 3 \mathrm{~h}$ & 88 & $91(R)$ \\
\hline 8 & CPME & 0.01 & 4a: $\mathrm{Cl}$ & r.t., $1 \mathrm{~h}$ & 87 & $91(R)$ \\
\hline 9 & DME & 0.01 & 4a: $\mathrm{Cl}$ & r.t., $15 \mathrm{~h}$ & N. R. & - \\
\hline 10 & TBME & 0.01 & 4b: $\mathrm{Br}$ & r.t., $1 \mathrm{~h}$ & 94 & $87(R)$ \\
\hline 11 & TBME & 0.01 & 4c: $\mathrm{H}$ & r.t., $24 \mathrm{~h}$ & 65 & $20(R)$ \\
\hline 12 & TBME & 0.01 & 4d: $\mathrm{Me}$ & r.t., $24 \mathrm{~h}$ & 99 & $25(R)$ \\
\hline 13 & TBME & 0.01 & 4e: $\mathrm{Ph}$ & r.t., $3 \mathrm{~h}$ & 79 & $18(R)$ \\
\hline
\end{tabular}

a) Catalyst concentration. $b$ ) Isolated yield. $c$ ) Determined by HPLC. $d$ ) The absolute configuration was determined by the comparison to literature value and authentic sample 3ca prepared from Michael adduct 3aa.

Table 2. Michael Reaction of Malonates with Various Acceptors

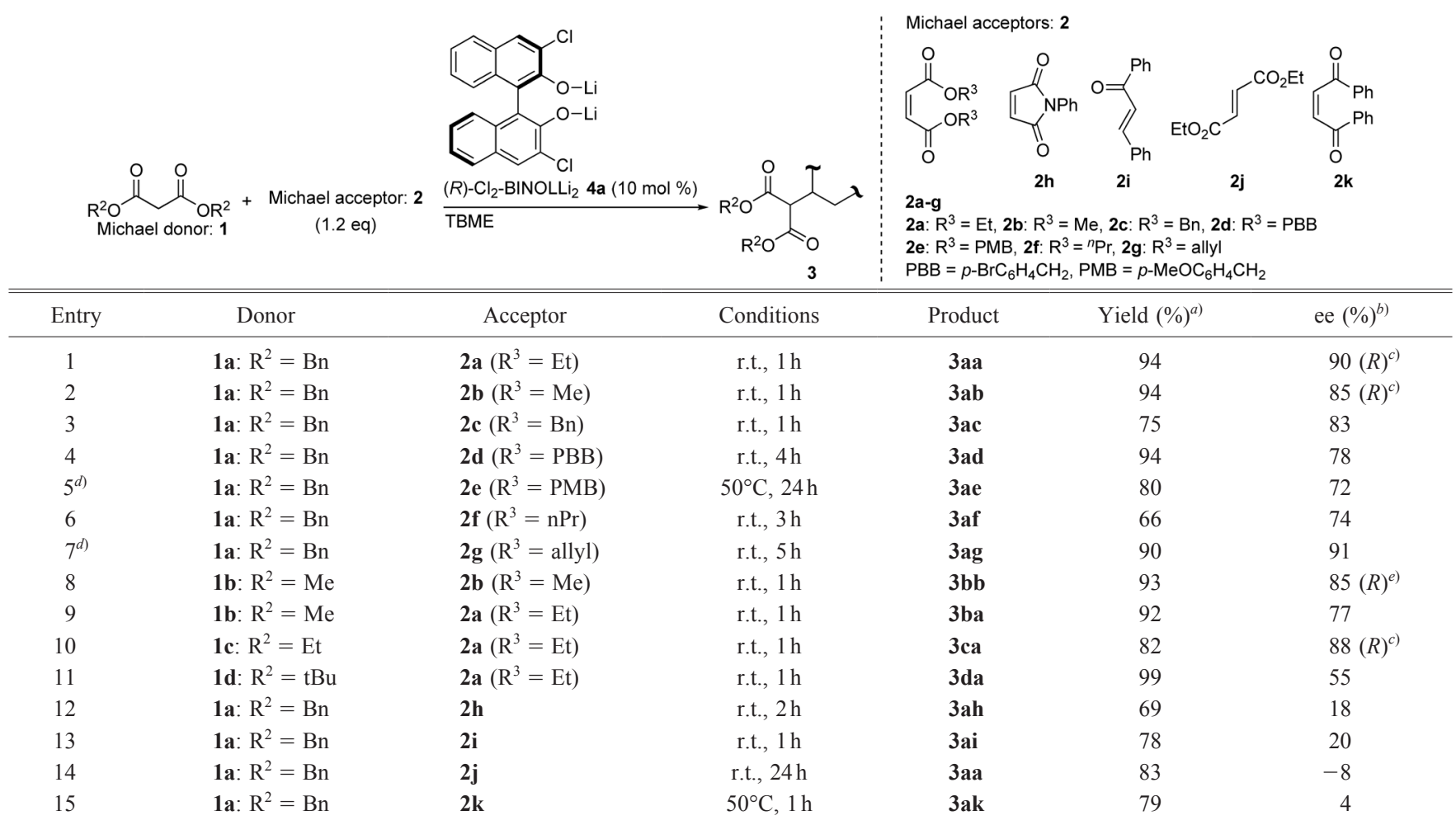

$a$ ) Isolated yield. $b$ ) Determined by HPLC. $c$ ) The absolute configuration was determined by the comparison to literature value and authentic sample $\mathbf{3 c a}$ prepared from each Michael adduct. $d)(R)-3,3^{\prime}-\mathrm{Cl}_{2}$-BINOL $(20 \mathrm{~mol} \%),{ }^{n} \mathrm{BuLi}(40 \mathrm{~mol} \%)$. $e$ ) The absolute configuration was determined to be $R$ by the comparison with the literature values of the HPLC retention time [CHIRALPAK AD-H, hexane-2-PrOH $(9: 1), 0.5 \mathrm{~mL} / \mathrm{min} ; 23.4 \mathrm{~min}(S), 24.32 \mathrm{~min}(R)]^{8)}$

geometry $(\mathbf{2 k})^{50)}$ yielded very low selectivity (entry 15 ).

Introduction of an additional substituent, such as a methyl, ethyl, benzyl, isopropyl, and halogen groups on the 2-position of malonates afforded adducts containing quaternary carbon (Table 3).

2-Alkyl malonate donors prepared the adducts 5ea, fa, fd, ga, ha, ic, ja, and kc with a sterically congested carbons in good yields with high enantioselectivities (entries 1-8), although 2-isopropyl malonates (1h and $\mathbf{1 i}$ ) required $20 \mathrm{~mol} \%$ catalyst and heating to $50^{\circ} \mathrm{C}$ to achieve a smooth reaction. This Michael reaction is not limited to 2-alkylmalonates. In the case of malonate bearing a chlorine atom (11), the elimina- 
Table 3. Michael Reaction of Substituted Malonates with Maleates

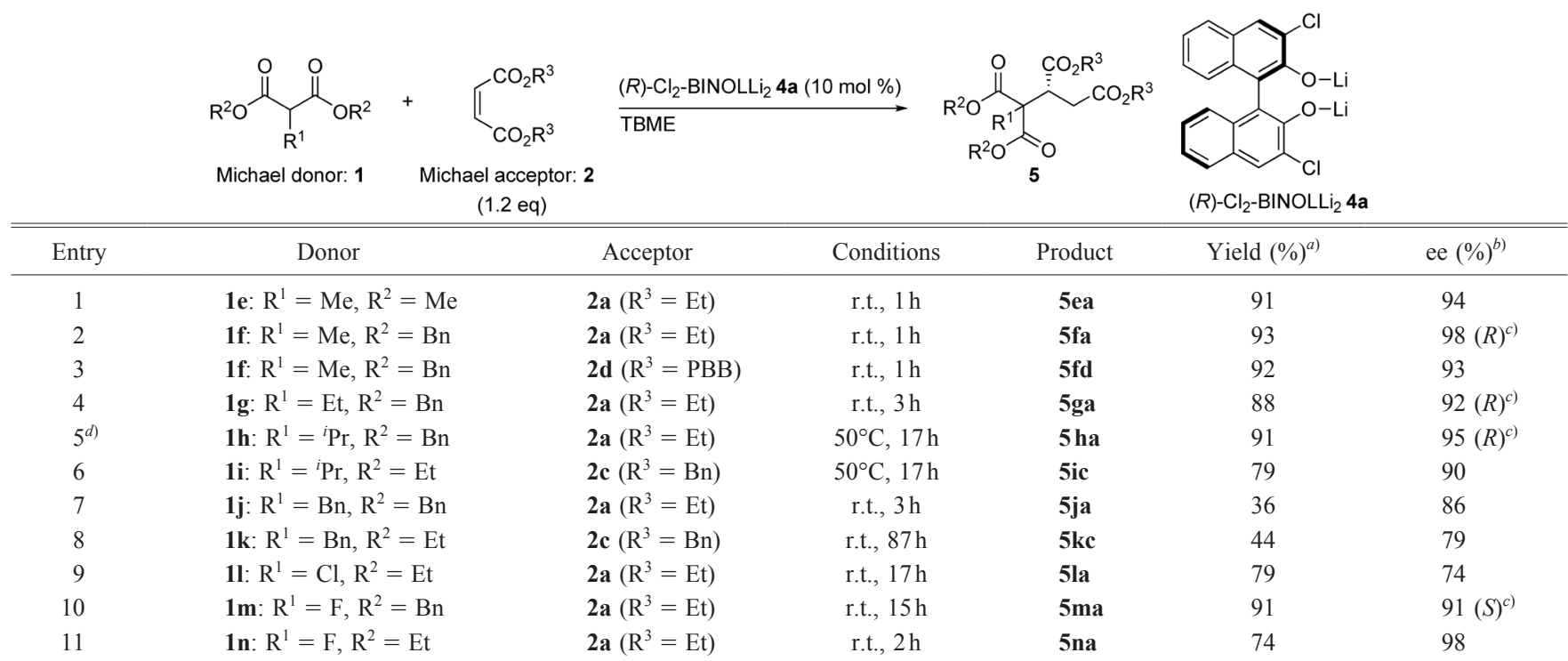

a) Isolated yield. $b$ ) Determined by HPLC. c) The absolute configuration was determined by the comparison with authentic samples prepared from the known compound $\mathbf{3}$ aa. d) $(R)-\mathrm{Cl}_{2}$-BINOL $(20 \mathrm{~mol} \%),{ }^{n} \mathrm{BuLi}(40 \mathrm{~mol} \%)$.

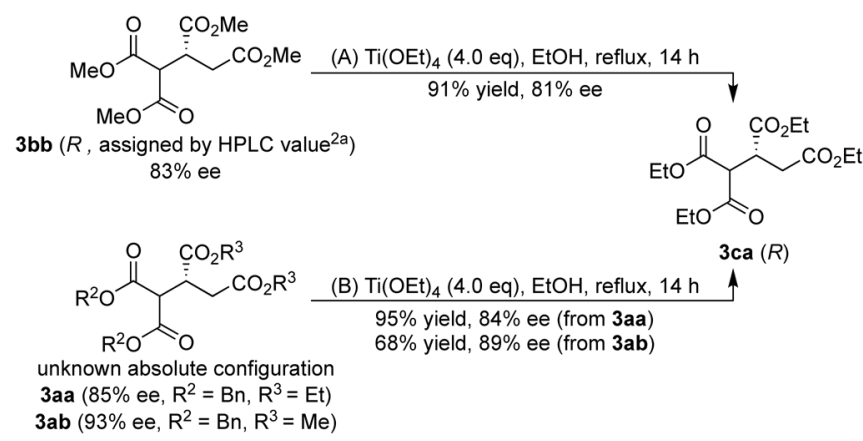

Chart 1. Determination of the Absolute Configurations of Compounds by Their Introduction into a Known Compound Using Transesterification

tion reaction proceeded from the adduct, resulting in a low yield of Michael adduct with moderate enantioselectivity (entry 9). However, 2-fluoro malonates $\mathbf{1 m}$ and $\mathbf{1 n}$ afforded the corresponding adducts in high yields with high enantioselectivities (entries 10, 11).

The absolute configuration of the Michael adduct $\mathbf{3 b b}$ prepared from $\mathbf{1 b}$ and $\mathbf{2 b}$ (Table 2, entry 8) was found to be $R$, which was assigned via the comparison with the HPLC data to the literature value. ${ }^{8)}$ Furthermore, methyl ester $\mathbf{3 b b}$ was converted to ethyl ester $\mathbf{3}$ ca by the transesterification reaction using $\mathrm{Ti}(\mathrm{OEt})_{4}$ (Chart 1A). The values of HPLC retention time from the two methods, transesterification product and Michael adduct prepared from $\mathbf{1 c}$ and $\mathbf{2 a}$ were identical. From these experiments, the absolute configuration of the obtained 3ca was determined to be $R$. Similarly, the absolute configuration of other adducts $\mathbf{3} \mathbf{a a}$ and $\mathbf{3} \mathbf{a b}$ were found to be $R$, after conversion to $\mathbf{3 c a}$ by transesterification (Chart 1B). As a result, it was found that the adducts $\mathbf{3} \mathbf{b b}$, 3aa, and $\mathbf{3} \mathbf{a b}$, respectively, have the same absolute configuration. It is suggested that the other Michael adducts possess the same configuration. Next, to determine the absolute configuration of compounds $\mathbf{5 f a}$, $5 \mathrm{ga}, 5 \mathrm{ha}$, and $\mathbf{5 m a}$, we conducted the functionalization for the known compound 3aa, which was prepared from $\mathbf{1 a}$ and

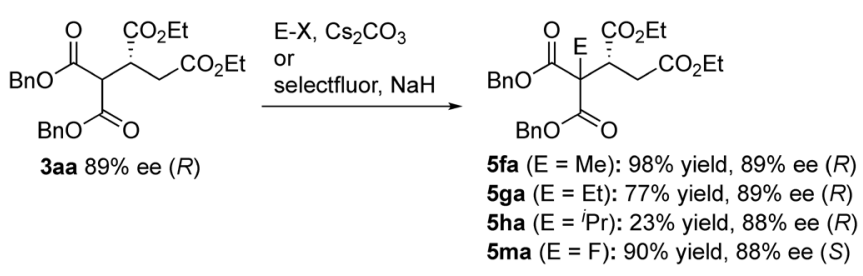

Chart 2. Determination of the Absolute Configurations of Compounds Bearing Quaternary Carbon Center
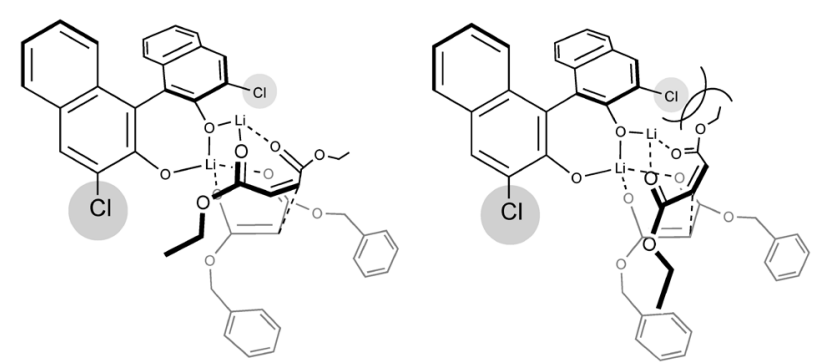

Fig. 1. Proposed Transition State in the Asymmetric Michael Reaction

2a (Table 1, entry 1). As shown in Chart 2, compound $\mathbf{5 f a}$ could be prepared from the obtained adduct 3aa by reacting with methyl iodide in the presence of base. ${ }^{51,52)}$ Comparing the HPLC value of this methylated product and Michael adduct from $\mathbf{1 f}$ and $\mathbf{2 a}$, the absolute configuration of $\mathbf{5 f a}$ was found to be $R$. Therefore, these results indicate that the adduct synthesized from 2-methyl benzyl malonate (1f) have the same absolute configuration as the adducts from unsubstituted malonate (1a). In addition, the absolute configurations of 5ga and 5ha were determined to be $R$ by the comparison with the values of the HPLC retention time after substitution reactions with appropriate alkyl halides in the presence of bases. Similarly, that of $\mathbf{5 m a}$ was determined to be $S$ after fluorination reaction using the Selectfluor.

Considering of the absolute configuration of products, the proposed transition state of this reaction is shown in Fig. 1. 


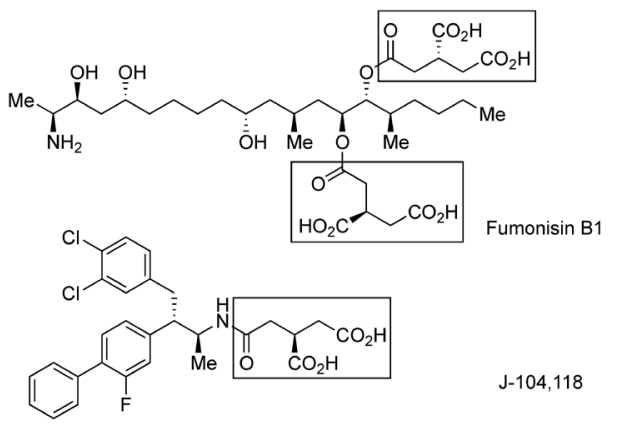

Fig. 2. Examples of Tricarboxylic Acid in Natural and Bioactive Compounds

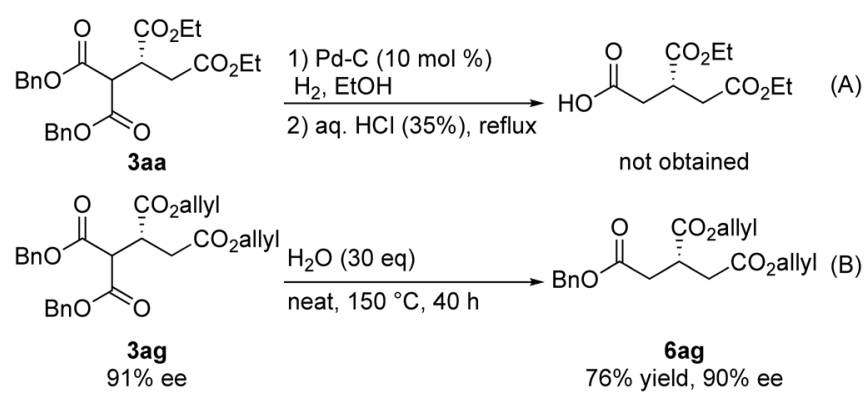

Chart 3. Decarboxylation of Tetracarboxylic Acid Ester

It is presumed that maleate ester approaches to the catalystbinding malonates while avoiding steric hindrance caused by the substitution of a catalyst, leading an enantioselective carbon-carbon bond formation.

Next, to demonstrate the utility of this reaction, the transformation of tetracarboxylic acid ester is accomplished. Optically active tricarboxylic acids (TCA) are widely found in biologically and pharmaceutically active compounds. Important natural compounds such as Fumonisin $\mathrm{B1}^{53-63)}$ or pharmaceutical compounds such as J-104, 118 ${ }^{64-67)}$ contain tricarboxylic acid moiety and possess bioactivities (Fig. 2).

For instance, in the reported methods for the synthesis of tricarboxylic acid moiety of Fumonisins or J-104, 118, enantioselective syntheses were achieved by using chiral auxiliary ${ }^{47,48,58,62)}$ or optical resolution of racemic compound using stoichiometric amount of chiral amines. ${ }^{64-66)}$ In order to improve the efficiency of the synthesis of such compounds, we envisioned that Michael reaction of malonates to maleates and subsequent decarboxylation would afford the desired tricarboxylic acid derivatives.

Our study for decarboxylation commenced with reaction under acidic media, ${ }^{68)}$ but the decarboxylation product was not obtained by heating with aqueous $\mathrm{HCl}$ after deprotection of benzyl group (Chart 3A). To improve this transformation, we performed the Krapcho reaction of $\mathbf{3 a g}$ in the presence of lithium salt, ${ }^{69-73)}$ but enantioselectivity of the product $\mathbf{6 a g}$ decreased. Finally, we discovered that on heating with water in a sealed tube in the absence of alkali bases ${ }^{74)}$ caused the decarboxylation reaction to proceed well, without the loss of remarkable enantioselectivity (Chart 3B). As mentioned above, these tricarboxylic acids and its derivatives are common and versatile structures in biologically active natural products. Our method allows facile access to the optically active tricarboxylic acid derivatives from commercially available materials through base-catalyzed Michael reaction.

In conclusion, we report the first example of enantioselective Michael reaction of malonates to maleates, catalyzed by dilithium 3,3'-dichlorobinaphtholate, affording the tetracarboxylic acid esters with high enantioselectivities. This Michael reaction can accommodate a wide range of donors and acceptors, even producing adducts with quaternary carbon. Furthermore, the resulting adduct can successfully be functionalized to biologically important compounds. Further investigation including the application to other substrates is ongoing in our laboratory.

\section{Experimental}

General All reactions were performed under argon atmosphere using dried glasswares equipped with a rubber septum and a magnetic stirring bar. The column chromatography purifications were performed using Kanto Chemical Silica Gel $60 \mathrm{~N}$ (spherical, neutral, 63-210 $\mu \mathrm{m}$ ). The IR spectra were recorded using a PerkinElmer, Inc. Frontier MIR/NIR. The ${ }^{1} \mathrm{H}-$ and ${ }^{13} \mathrm{C}$-NMR spectra were recorded in $\mathrm{CDCl}_{3}$ using a JEOL JNM-ECX-400 spectrometer. The chemical shifts are reported in ppm relative to the internal tetramethylsilane (TMS) standard $(\delta 0.00 \mathrm{ppm})$ for the ${ }^{1} \mathrm{H}-\mathrm{NMR}$ spectra and solvent signals $(\delta 77.0 \mathrm{ppm})$ for the ${ }^{13} \mathrm{C}-\mathrm{NMR}$ spectra. The mass spectra were recorded using a JEOL JMS-700MStation mass spectrometer. The HPLC analyses were performed using JASCO P-2080 and UV-2075.

Typical Procedure of Michael Reaction (Table 1, Entry 5) Under argon atmosphere, $n$-butyllithium $(0.10 \mathrm{mmol}$, $20 \mathrm{~mol} \%)$ in hexane $(0.15 \mathrm{M}, 0.67 \mathrm{~mL})$ was added to a solution of $(R)-3,3^{\prime}-\mathrm{Cl}_{2}$-BINOL $(17.8 \mathrm{mg}, \quad 0.05 \mathrm{mmol}, \quad 10 \mathrm{~mol} \%)$ in TBME $(5.0 \mathrm{~mL})$ at $0^{\circ} \mathrm{C}$. After stirring for $1 \mathrm{~min}$, dibenzyl malonate (1a) $(0.125 \mathrm{~mL}, 0.5 \mathrm{mmol}, 1.0 \mathrm{eq})$ and diethyl maleate (2a) $(0.096 \mathrm{~mL}, 0.6 \mathrm{mmol}, 1.2 \mathrm{eq})$ was successively added to the mixture at room temperature (r.t.). After $1 \mathrm{~h}$, the reaction was quenched with sat. $\mathrm{NH}_{4} \mathrm{Cl}$ aq. $(2 \mathrm{~mL})$ and stirred for $0.5 \mathrm{~h}$. The aqueous layer was extracted with EtOAc $(3 \times 10 \mathrm{~mL})$. The combined organic layers were washed with brine $(20 \mathrm{~mL})$, and dried over $\mathrm{Na}_{2} \mathrm{SO}_{4}$. After filtration and concentration, the crude product was purified by column chromatography (hexane-EtOAc $=9: 1, \mathrm{SiO}_{2}: 10 \mathrm{~g}$ ) to give product $\mathbf{3 a a}$ as a colorless oil (214 mg, 94\% yield, 90\% ee).

(R)-1,1-Dibenzyl 2,3-Diethyl Propane-1,1,2,3-tetracarboxylate (3aa) ${ }^{46)}$ Colorless oil. $214 \mathrm{mg}$. 94\% yield. TLC: $R f$ 0.52 (hexane-EtOAc $=4: 1$, stained white with anisaldehyde). $[\alpha]_{435}^{27}+11.5\left(c=1.03, \mathrm{CHCl}_{3}\right)$ for $90 \%$ ee. ${ }^{1} \mathrm{H}-\mathrm{NMR}\left(\mathrm{CDCl}_{3}\right)$ $\delta: 1.15(3 \mathrm{H}, \mathrm{t}, J=7.2 \mathrm{~Hz}), 1.23(3 \mathrm{H}, \mathrm{t}, J=7.6 \mathrm{~Hz}), 2.66(1 \mathrm{H}$, dd, $J=17.0,5.2 \mathrm{~Hz}), 2.79(1 \mathrm{H}, \mathrm{dd}, J=17.0,7.6 \mathrm{~Hz}), 3.60-3.62$ $(1 \mathrm{H}, \mathrm{m}), 4.04-4.12(5 \mathrm{H}, \mathrm{m}), 5.14(2 \mathrm{H}, \mathrm{s}), 5.15(2 \mathrm{H}, \mathrm{s}), 7.28-7.37$ $(10 \mathrm{H}, \mathrm{m})$. The enantiomeric excess was determined to be $90 \%$ ee by chiral HPLC with Daicel Chiralpak AD-H column [eluent: hexane-IPA $=9: 1$; flow rate: $1.0 \mathrm{~mL} / \mathrm{min}$; detection: $\left.254 \mathrm{~nm} ; t_{\mathrm{R}}: 22.0 \mathrm{~min}(R), 23.7 \mathrm{~min}(S)\right]$.

(R)-1,1-Dibenzyl 2,3-Dimethyl Propane-1,1,2,3-tetracarboxylate (3ab) ${ }^{46)}$ Colorless oil. $201 \mathrm{mg}$. 94\% yield. TLC: $R f$ 0.41 (hexane-EtOAc $=4: 1$, stained blue with phosphomolybdic acid). $[\alpha]_{435}^{29}+7.5\left(c=0.88, \mathrm{CHCl}_{3}\right)$ for $85 \%$ ee. ${ }^{1} \mathrm{H}-\mathrm{NMR}$ $\left(\mathrm{CDCl}_{3}\right) \delta: 2.67(1 \mathrm{H}, \mathrm{dd}, J=17.0,4.8 \mathrm{~Hz}), 2.80(1 \mathrm{H}, \mathrm{dd}$, $J=17.0,8.0 \mathrm{~Hz}), 3.58(3 \mathrm{H}, \mathrm{s}), 3.60-3.64(1 \mathrm{H}, \mathrm{m}), 3.65(3 \mathrm{H}, \mathrm{s})$, $4.03(1 \mathrm{H}, \mathrm{d}, J=6.8 \mathrm{~Hz}), 5.15(2 \mathrm{H}, \mathrm{s}), 5.16(2 \mathrm{H}, \mathrm{s}), 7.26-7.37$ $(10 \mathrm{H}, \mathrm{m})$. The enantiomeric excess was determined to be 
$85 \%$ ee by chiral HPLC with Daicel Chiralpak AD-3 column [eluent: hexane-IPA $=19: 1$; flow rate: $1.0 \mathrm{~mL} / \mathrm{min}$; detection: $\left.254 \mathrm{~nm} ; t_{\mathrm{R}}: 54.0 \mathrm{~min}(R), 56.9 \mathrm{~min}(S)\right]$.

(+)-Tetrabenzyl Propane-1,1,2,3-tetracarboxylate (3ac) ${ }^{46)}$ Colorless oil. $217 \mathrm{mg}$. $75 \%$ yield. TLC: $R f 0.33$ (hexaneEtOAc $=4: 1$, stained blue with phosphomolybdic acid). $[\alpha]_{435}^{31}$ $+11.4\left(c=1.15, \mathrm{CHCl}_{3}\right)$ for $83 \%$ ee. ${ }^{1} \mathrm{H}-\mathrm{NMR}\left(\mathrm{CDCl}_{3}\right) \delta$ : $2.72(1 \mathrm{H}, \mathrm{dd}, J=17.0,5.2 \mathrm{~Hz}), 2.88(1 \mathrm{H}, \mathrm{dd}, J=17.0,7.6 \mathrm{~Hz})$, $3.67-3.70(1 \mathrm{H}, \mathrm{m}), 4.05(1 \mathrm{H}, \mathrm{d}, J=6.8 \mathrm{~Hz}), 5.00-5.08(8 \mathrm{H}, \mathrm{m})$, $7.21-7.34(20 \mathrm{H}, \mathrm{m})$. The enantiomeric excess was determined to be $83 \%$ ee by chiral HPLC with Daicel Chiralpak AS-H column [eluent: hexane-IPA $=4: 1$; flow rate: $1.0 \mathrm{~mL} / \mathrm{min}$; detection: $254 \mathrm{~nm} ; t_{\mathrm{R}}: 11.5 \mathrm{~min}$ (minor), $15.0 \mathrm{~min}$ (major)].

(+)-1,1-Dibenzyl 2,3-Bis(4-bromobenzyl) Propane-1,1,2,3tetracarboxylate (3ad) Pale yellow oil. $346 \mathrm{mg}$. 94\% yield. TLC: Rf 0.47 (hexane-EtOAc $=4: 1$, stained white with anisaldehyde). $[\alpha]_{435}^{19}+5.8\left(c=1.2, \mathrm{CHCl}_{3}\right)$ for $78 \%$ ee. IR (ATR) $\mathrm{cm}^{-1}: 3035,1729,1153 .{ }^{1} \mathrm{H}-\mathrm{NMR}\left(\mathrm{CDCl}_{3}\right) \delta: 2.71(1 \mathrm{H}$, dd, $J=16.9,4.6 \mathrm{~Hz}), 2.86(1 \mathrm{H}, \mathrm{dd}, J=16.9,7.8 \mathrm{~Hz}), 3.65-3.70$ $(1 \mathrm{H}, \mathrm{m}), 4.00-4.03(1 \mathrm{H}, \mathrm{m}), 4.94(2 \mathrm{H}, \mathrm{s}), 4.98(2 \mathrm{H}, \mathrm{s}), 5.04$ $(2 \mathrm{H}, \mathrm{s}), 5.09(2 \mathrm{H}, \mathrm{s}), 7.07(2 \mathrm{H}, \mathrm{d}, J=8.2 \mathrm{~Hz}), 7.14(2 \mathrm{H}, \mathrm{d}$, $J=8.2 \mathrm{~Hz}), 7.22-7.31(10 \mathrm{H}, \mathrm{m}), 7.41(2 \mathrm{H}, \mathrm{d}, J=8.2 \mathrm{~Hz}), 7.45$ $(2 \mathrm{H}, \mathrm{d}, J=7.8 \mathrm{~Hz}) .{ }^{13} \mathrm{C}-\mathrm{NMR}\left(\mathrm{CDCl}_{3}\right) \delta: 33.2,40.3,52.1,66.4$, $65.8,67.5,67.6,122.3,122.3,128.2,128.4,128.4,128.5,129.8$, $129.9,131.6,131.6,134.1,134.5,134.7,134.8,167.1,167.3$, 170.7, 171.1 (two carbons overlapped). FAB-MS m/z: 759.0211 (Calcd for $\mathrm{C}_{35} \mathrm{H}_{30} \mathrm{Br}_{2} \mathrm{O}_{8} \mathrm{Na}$ : 761.0188). MS m/z: 759, 761, 763 $\left(\mathrm{M}+\mathrm{Na}^{+}\right)$, 91. The enantiomeric excess was determined to be $78 \%$ ee by chiral HPLC with Daicel Chiralcel AS-H column [eluent: hexane-IPA $=4: 1$; flow rate: $1.0 \mathrm{~mL} / \mathrm{min}$; detection: $254 \mathrm{~nm} ; t_{\mathrm{R}}: 24.3 \mathrm{~min}$ (minor), $32.2 \mathrm{~min}$ (major)].

(+)-1,1-Dibenzyl 2,3-Bis(4-methoxybenzyl) Propane1,1,2,3-tetracarboxylate (3ae) Colorless oil. $256 \mathrm{mg}$. $80 \%$ yield. TLC: $R f 0.44$ (hexane-EtOAc $=4: 1$, stained white with anisaldehyde). $[\alpha]_{435}^{19}+9.5\left(c=0.92, \mathrm{CHCl}_{3}\right)$ for $72 \%$ ee. IR (ATR) $\mathrm{cm}^{-1}: 3034,1732,1153 .{ }^{1} \mathrm{H}-\mathrm{NMR}\left(\mathrm{CDCl}_{3}\right) \delta: 2.68(1 \mathrm{H}$, $\mathrm{dd}, J=16.9,5.0 \mathrm{~Hz}), 2.83(1 \mathrm{H}, \mathrm{dd}, J=16.9,8.2 \mathrm{~Hz}), 3.63-3.68$ $(1 \mathrm{H}, \mathrm{m}), 3.77(3 \mathrm{H}, \mathrm{s}), 3.80(3 \mathrm{H}, \mathrm{s}), 4.02(1 \mathrm{H}, \mathrm{d}, J=6.9 \mathrm{~Hz})$, 4.93-4.98 (4H, m), $5.02(2 \mathrm{H}, \mathrm{s}), 5.07(2 \mathrm{H}, \mathrm{s}), 6.81(2 \mathrm{H}, \mathrm{d}$, $J=8.7 \mathrm{~Hz}), 6.85(2 \mathrm{H}, \mathrm{d}, J=8.2 \mathrm{~Hz}), 7.16(2 \mathrm{H}, \mathrm{d}, J=8.7 \mathrm{~Hz})$, 7.21-7.31 (12H, m). ${ }^{13} \mathrm{C}-\mathrm{NMR}\left(\mathrm{CDCl}_{3}\right) \delta: 33.4,40.5,52.2,55.3$, $66.5,67.1,67.5,67.5,113.8,113.9,127.3,127.7,128.2,128.4$, $128.5,130.1,130.2,134.9,135.0,159.6,159.6,167.3,167.4$, 171.0, 171.3 (four carbons overlapped). FAB-MS $m / z: 663.2218$ (Calcd for $\mathrm{C}_{37} \mathrm{H}_{36} \mathrm{O}_{10} \mathrm{Na}:$ 663.2206). MS m/z: $663\left(\mathrm{M}+\mathrm{Na}^{+}\right)$, 91. The enantiomeric excess was determined to be $72 \%$ ee by chiral HPLC with Daicel Chiralcel AD-3 column [eluent: hexane-IPA $=4: 1$; flow rate: $1.0 \mathrm{~mL} / \mathrm{min}$; detection: $254 \mathrm{~nm}$; $t_{\mathrm{R}}: 47.6 \mathrm{~min}$ (major), $51.4 \mathrm{~min}$ (minor)].

(+)-1,1-Dibenzyl 2,3-Dipropyl Propane-1,1,2,3-tetracarboxylate (3af) Colorless oil. $160 \mathrm{mg}$. 66\% yield. TLC: $R f$ 0.26 (hexane-EtOAc $=4: 1$, stained blue with phosphomolybdic acid). $[\alpha]_{435}^{27}+11.5\left(c=1.07, \mathrm{CHCl}_{3}\right)$ for $74 \%$ ee. IR (ATR) $\mathrm{cm}^{-1}: 3035,2967,1730,1151 .{ }^{1} \mathrm{H}-\mathrm{NMR}\left(\mathrm{CDCl}_{3}\right) \delta: 0.86$ $(3 \mathrm{H}, \mathrm{t}, J=7.4 \mathrm{~Hz}), 0.91(3 \mathrm{H}, \mathrm{t}, J=7.3 \mathrm{~Hz}), 1.50-1.66(4 \mathrm{H}, \mathrm{m})$, $2.67(1 \mathrm{H}, \mathrm{dd}, J=17.0,4.6 \mathrm{~Hz}), 2.81(1 \mathrm{H}, \mathrm{dd}, J=17.0,7.8 \mathrm{~Hz})$, 3.60-3.62 (1H, m), 4.04-4.12 (5H, m), $5.14(2 \mathrm{H}, \mathrm{s}), 5.15(2 \mathrm{H}$, s), 7.28-7.37 (10H, m) ${ }^{13} \mathrm{C}-\mathrm{NMR}\left(\mathrm{CDCl}_{3}\right) \delta: 10.2,10.3,21.7$, $21.8,33.4,40.5,52.3,66.4,67.0,67.5,128.2,128.4,128.4$, $128.5,135.0,135.0,167.3,167.5,171.1,171.5$ (three carbons overlapped). FAB-MS m/z: 485.2173 (Calcd for $\mathrm{C}_{27} \mathrm{H}_{33} \mathrm{O}_{8}$ :
485.2175). MS $m / z: 485\left(\mathrm{M}+\mathrm{H}^{+}\right), 91$. The enantiomeric excess was determined to be $74 \%$ ee by chiral HPLC with Daicel Chiralpak AD-H column [eluent: hexane-IPA = 9: 1 ; flow rate: $1.0 \mathrm{~mL} / \mathrm{min}$; detection: $254 \mathrm{~nm} ; t_{\mathrm{R}}: 15.9 \mathrm{~min}$ (major), $17.4 \mathrm{~min}$ (minor)].

(+)-2,3-Diallyl 1,1-Dibenzyl Propane-1,1,2,3-tetracarboxylate (3ag) Colorless oil. $216 \mathrm{mg}$. 90\% yield. TLC: $R f$ 0.47 (hexane-EtOAc $=4: 1$, stained purple with anisaldehyde). $[\alpha]_{435}^{19}+7.8\left(c=0.98, \mathrm{CHCl}_{3}\right)$ for $91 \%$ ee. IR (ATR) $\mathrm{cm}^{-1}: 3034$, 1731, 1649, 1153. ${ }^{1} \mathrm{H}-\mathrm{NMR}\left(\mathrm{CDCl}_{3}\right) \delta: 2.72(1 \mathrm{H}, \mathrm{dd}, J=17.2$, $4.6 \mathrm{~Hz}), 2.85(1 \mathrm{H}, \mathrm{dd}, J=17.2,7.8 \mathrm{~Hz}), 3.64-3.69(1 \mathrm{H}, \mathrm{m})$, $4.05(1 \mathrm{H}, \mathrm{d}, J=6.9 \mathrm{~Hz}), 4.51(2 \mathrm{H}, \mathrm{d}, J=6.0 \mathrm{~Hz}), 4.55(2 \mathrm{H}$, $\mathrm{d}, J=6.0 \mathrm{~Hz}), 5.14(2 \mathrm{H}, \mathrm{s}), 5.15(2 \mathrm{H}, \mathrm{s}), 5.17-5.32(4 \mathrm{H}, \mathrm{m})$, 5.74-5.91 (2H, m), 7.26-7.32 (10H, m). ${ }^{13} \mathrm{C}-\mathrm{NMR}\left(\mathrm{CDCl}_{3}\right) \delta$ : $33.2,40.4,52.2,65.5,66.1,67.6,118.4,118.6,128.3,128.4$, $128.4,128.5,131.5,131.8,134.9,135.0,167.3,167.4,170.7$, 171.1 (three carbons overlapped). FAB-MS $\mathrm{m} / \mathrm{z}: 481.1892$ (Calcd for $\mathrm{C}_{27} \mathrm{H}_{29} \mathrm{O}_{8}$ : 481.1862). MS $m / z$ : $481\left(\mathrm{M}+\mathrm{H}^{+}\right.$). The enantiomeric excess was determined to be $91 \%$ ee by chiral HPLC with Daicel Chiralpak AS-H column [eluent: hexane-IPA $=9: 1$; flow rate: $1.0 \mathrm{~mL} / \mathrm{min}$; detection: $254 \mathrm{~nm} ; t_{\mathrm{R}}$ : 16.6 min (minor), 21.7 min (major)].

(R)-Tetramethyl Propane-1,1,2,3-tetracarboxylate (3bb) $)^{8)}$ Colorless oil. $127 \mathrm{mg}$. 93\% yield. TLC: $R f 0.26$ (hexaneEtOAc $=4: 1$, stained blue with phosphomolybdic acid). $[\alpha]_{\mathrm{D}}^{29}$ $+13.4\left(c=0.2, \mathrm{CHCl}_{3}\right)$ for $85 \%$ ee $(R)$. $\left[\left(\mathrm{lit}^{8)}:[\alpha]_{\mathrm{D}}^{25}+5.3\right.\right.$, $\left.c=0.2, \mathrm{CHCl}_{3}\right)$ for $93 \%$ ee $\left.(R)\right] .{ }^{1} \mathrm{H}-\mathrm{NMR}\left(\mathrm{CDCl}_{3}\right) \delta: 2.72$ $(1 \mathrm{H}, \mathrm{dd}, J=17.2,4.8 \mathrm{~Hz}), 2.84(1 \mathrm{H}, \mathrm{dd}, J=17.2,7.0 \mathrm{~Hz})$, $3.58-3.63(1 \mathrm{H}, \mathrm{m}), 3.70(3 \mathrm{H}, \mathrm{s}), 3.72(3 \mathrm{H}, \mathrm{s}), 3.76(3 \mathrm{H}, \mathrm{s}), 3.78$ $(3 \mathrm{H}, \mathrm{s}), 3.94(1 \mathrm{H}, \mathrm{d}, J=6.8 \mathrm{~Hz})$. The enantiomeric excess was determined to be $85 \%$ ee by chiral HPLC with Daicel Chiralpak AD-H column [eluent: hexane-IPA $=9: 1$; flow rate: $0.5 \mathrm{~mL} / \mathrm{min}$; detection: $\left.220 \mathrm{~nm} ; t_{\mathrm{R}}: 23.4 \mathrm{~min}(S), 24.3 \mathrm{~min}(R)\right]$.

(+)-2,3-Diethyl 1,1-Dimethyl Propane-1,1,2,3-tetracarboxylate (3ba) ${ }^{46)}$ Pale yellow oil. $140 \mathrm{mg}$. 92\% yield. TLC: Rf 0.71 (hexane-EtOAc $=1: 1$, stained blue with phosphomolybdic acid). $[\alpha]_{435}^{28}+29.6\left(c=1.02, \mathrm{CHCl}_{3}\right)$ for $77 \%$ ee. ${ }^{1} \mathrm{H}-\mathrm{NMR}\left(\mathrm{CDCl}_{3}\right) \delta: 1.24(3 \mathrm{H}, \mathrm{t}, J=7.4 \mathrm{~Hz}), 1.32(3 \mathrm{H}, \mathrm{t}$, $J=7.4 \mathrm{~Hz}), 2.69(1 \mathrm{H}, \mathrm{dd}, J=16.8,5.2 \mathrm{~Hz}), 2.81(1 \mathrm{H}, \mathrm{dd}$, $J=16.8,8.0 \mathrm{~Hz}), 3.56-3.61(1 \mathrm{H}, \mathrm{m}), 3.75(3 \mathrm{H}, \mathrm{s}), 3.76(3 \mathrm{H}, \mathrm{s})$, $3.94(1 \mathrm{H}, \mathrm{d}, J=7.6 \mathrm{~Hz}), 4.15(2 \mathrm{H}, \mathrm{q}, J=7.4 \mathrm{~Hz}), 4.17(2 \mathrm{H}, \mathrm{q}$, $J=7.4 \mathrm{~Hz}$ ). The enantiomeric excess was determined to be $77 \%$ ee by chiral HPLC with Daicel Chiralpak AD-3 column [eluent: hexane-IPA $=29: 1$; flow rate: $1.0 \mathrm{~mL} / \mathrm{min}$; detection: $220 \mathrm{~nm} ; t_{\mathrm{R}}: 27.6 \mathrm{~min}$ (minor), $28.6 \mathrm{~min}$ (major)].

(+)-Tetraethyl Propane-1,1,2,3-tetracarboxylate (3ca) Pale yellow oil. $136 \mathrm{mg}$. $82 \%$ yield. TLC: Rf 0.26 (hexaneEtOAc $=4: 1$, stained blue with phosphomolybdic acid). $[\alpha]_{435}^{29}$ $+23.2\left(c=1.01, \mathrm{CHCl}_{3}\right)$ for $88 \%$ ee. IR (film on $\left.\mathrm{NaCl}\right) \mathrm{cm}^{-1}$ : 2983, 1731, 1164. ${ }^{1} \mathrm{H}-\mathrm{NMR}\left(\mathrm{CDCl}_{3}\right) \delta: 1.24-1.29(12 \mathrm{H}, \mathrm{m})$, $2.69(1 \mathrm{H}, \mathrm{dd}, J=16.8,5.2 \mathrm{~Hz}), 2.81(1 \mathrm{H}, \mathrm{dd}, J=16.8,8.0 \mathrm{~Hz})$, $3.55-3.60$ (1H, m), 3.89 (1H, d, $J=7.2 \mathrm{~Hz}), 4.12-4.25(8 \mathrm{H}, \mathrm{m})$. ${ }^{13} \mathrm{C}-\mathrm{NMR}\left(\mathrm{CDCl}_{3}\right) \delta$ : 14.0, 14.1, 33.5, 40.5, 52.4, 60.8, 61.4, 61.8, 167.7, 167.8, 171.2, 171.7 (three carbons overlapped). FAB-MS m/z: 333.1559 (Calcd for $\mathrm{C}_{15} \mathrm{H}_{25} \mathrm{O}_{8}$ : 333.1549). MS $m / z$ : $333\left(\mathrm{M}+\mathrm{H}^{+}\right), 259$. The enantiomeric excess was determined to be $88 \%$ ee by chiral HPLC with Daicel Chiralpak AS-H column [eluent: hexane-IPA $=49: 1$; flow rate: $1.0 \mathrm{~mL} / \mathrm{min}$; detection: $220 \mathrm{~nm}$; $t_{\mathrm{R}}: 17.1 \mathrm{~min}$ (minor), $18.7 \mathrm{~min}$ (major)].

(+)-1,1-Di-tert-butyl 2,3-Diethyl Propane-1,1,2,3-tetra- 
carboxylate (3da) ${ }^{46)}$ Colorless oil. $192 \mathrm{mg} . \quad 99 \%$ yield. TLC: Rf 0.29 (hexane-EtOAc $=4: 1$, stained blue with phosphomolybdic acid). $[\alpha]_{435}^{29}+11.5\left(c=1.13, \mathrm{CHCl}_{3}\right)$ for $55 \%$ ee. ${ }^{1} \mathrm{H}-\mathrm{NMR}\left(\mathrm{CDCl}_{3}\right) \delta$ : $1.23-1.28(6 \mathrm{H}, \mathrm{m}), 1.46(18 \mathrm{H}, \mathrm{s}$, $18 \mathrm{H}), 2.69(1 \mathrm{H}, \mathrm{dd}, J=16.8,4.4 \mathrm{~Hz}), 2.78(1 \mathrm{H}, \mathrm{dd}, J=16.8$, $8.4 \mathrm{~Hz}), 3.45-3.50(1 \mathrm{H}, \mathrm{m}), 3.71(1 \mathrm{H}, \mathrm{d}, J=7.2 \mathrm{~Hz}), 4.14(2 \mathrm{H}$, $\mathrm{q}, J=7.6 \mathrm{~Hz}), 4.18(2 \mathrm{H}, \mathrm{q}, J=7.2 \mathrm{~Hz})$. The enantiomeric excess was determined to be $55 \%$ ee by chiral HPLC with Daicel Chiralpak AD-H column [eluent: hexane-IPA = 29: ; flow rate: $1.0 \mathrm{~mL} / \mathrm{min}$; detection: $220 \mathrm{~nm} ; t_{\mathrm{R}}: 9.7 \mathrm{~min}$ (minor), 11.4 min (major)].

(-)-Dibenzyl 2-(2,5-Dioxo-1-phenylpyrrolidin-3-yl)malonate $(\mathbf{3 a h})^{46)}$ Colorless prisms. mp: $90.0-92.0^{\circ} \mathrm{C} .158 \mathrm{mg}$. $69 \%$ yield. TLC: $R f 0.66$ (hexane-EtOAc $=1: 1$, stained blue with phosphomolybdic acid). $[\alpha]_{435}^{30}-5.8\left(c=0.94, \mathrm{CHCl}_{3}\right)$ for $18 \%$ ee. ${ }^{1} \mathrm{H}-\mathrm{NMR}\left(\mathrm{CDCl}_{3}\right) \quad \delta: 2.88-3.00(2 \mathrm{H}, \mathrm{m}), 3.45-3.51$ $(1 \mathrm{H}, \mathrm{m}), 4.31(1 \mathrm{H}, \mathrm{d}, J=4.0 \mathrm{~Hz}), 5.18(2 \mathrm{H}, \mathrm{s}), 5.21(2 \mathrm{H}, \mathrm{s})$, $7.19(2 \mathrm{H}, \mathrm{d}, J=7.6 \mathrm{~Hz}), 7.26-7.45(13 \mathrm{H}, \mathrm{m})$. The enantiomeric excess was determined to be $18 \%$ ee by chiral HPLC with Daicel Chiralcel OZ-H column [eluent: hexane-IPA $=1: 1$; flow rate: $1.0 \mathrm{~mL} / \mathrm{min}$; detection: $254 \mathrm{~nm} ; t_{\mathrm{R}}: 19.5 \mathrm{~min}$ (minor), $40.0 \mathrm{~min}$ (major)].

(S)-Dibenzyl 2-(3-Oxo-1,3-diphenylpropyl)malonate (3ai) ${ }^{10)}$ Colorless oil. $192 \mathrm{mg}$. $78 \%$ yield. TLC: $R f 0.51$ (hexaneEtOAc $=4: 1$, stained blue with anisaldehyde). $[\alpha]_{\mathrm{D}}^{25}+1.6$ $\left(c=1.0, \mathrm{CHCl}_{3}\right)$ for $20 \%$ ee. $\left[\left(\mathrm{lit}^{10)}:[\alpha]_{\mathrm{D}}^{25}+18.4\left(c=1.0, \mathrm{CHCl}_{3}\right)\right.\right.$ for $91 \%$ ee $(S)]$. ${ }^{1} \mathrm{H}-\mathrm{NMR}\left(\mathrm{CDCl}_{3}\right) \delta: 3.44(2 \mathrm{H}, \mathrm{d}, J=6.8 \mathrm{~Hz})$, $3.94(1 \mathrm{H}, \mathrm{d}, J=9.8 \mathrm{~Hz}), 4.19-4.25(1 \mathrm{H}, \mathrm{m}), 4.90(2 \mathrm{H}, \mathrm{s}), 5.12$ $(1 \mathrm{H}, \mathrm{d}, J=12.4 \mathrm{~Hz}), 5.17(1 \mathrm{H}, \mathrm{d}, J=12.4 \mathrm{~Hz}), 7.05-7.07(2 \mathrm{H}$, m), 7.15-7.34 (13H, m), 7.36-7.43 (2H, m), 7.46-7.55 (1H, m), $7.80(2 \mathrm{H}, \mathrm{d}, J=8.2 \mathrm{~Hz})$. The ee was determined to be $20 \%$ ee by chiral HPLC with Daicel Chiralpak AS-H column [eluent: hexane-IPA $=9: 1$; flow rate: $1.0 \mathrm{~mL} / \mathrm{min}$; detection: $254 \mathrm{~nm}$; $\left.t_{\mathrm{R}}: 19.9 \min (R), 23.0 \min (S)\right]$.

(S)-1,1-Dibenzyl 2,3-Diethyl Propane-1,1,2,3-tetracarboxylate (3aa) ${ }^{46)}$ Colorless oil. $189 \mathrm{mg}$. $83 \%$ yield. TLC: $R f$ 0.52 (hexane-EtOAc $=4: 1$, stained white with anisaldehyde). $[\alpha]_{435}^{29}-1.1\left(c=1.1, \mathrm{CHCl}_{3}\right)$ for $-8 \%$ ee. The enantiomeric excess was determined to be $-8 \%$ ee by chiral HPLC with Daicel Chiralpak AD-H column [eluent: hexane-IPA $=9: 1$; flow rate: $1.0 \mathrm{~mL} / \mathrm{min}$; detection: $254 \mathrm{~nm} ; t_{\mathrm{R}}: 20.8 \mathrm{~min}(R)$, $22.2 \mathrm{~min}(S)]$.

(S)-Dibenzyl 2-(1,4-Dioxo-1,4-diphenylbutan-2-yl)malonate (3ak) ${ }^{50)}$ White prisms. mp: $66.0-68.0^{\circ}$ C. $205 \mathrm{mg} .79 \%$ yield. TLC: $R f 0.50$ (hexane-EtOAc $=4: 1$, stained blue with anisaldehyde). $[\alpha]_{\mathrm{D}}^{24}+1.1\left(c=1.1, \mathrm{CHCl}_{3}\right)$ for $4 \%$ ee. $\left[\left(\mathrm{lit}^{50)}\right.\right.$ : $[\alpha]_{\mathrm{D}}^{23}-23.4\left(c=0.25, \mathrm{CHCl}_{3}\right)$ for $88 \%$ ee $\left.(R)\right] ;{ }^{1} \mathrm{H}-\mathrm{NMR}$ $\left(\mathrm{CDCl}_{3}\right) \delta: 3.38(1 \mathrm{H}, \mathrm{dd}, J=18.3,6.3 \mathrm{~Hz}), 3.54(1 \mathrm{H}, \mathrm{dd}$, $J=18.3,5.9 \mathrm{~Hz}), 4.04(1 \mathrm{H}, \mathrm{d}, J=8.4 \mathrm{~Hz}), 4.9-5.13(5 \mathrm{H}, \mathrm{m})$, 7.14-7.28 (10H, m), 7.35-7.44 (4H, m), 7.52-7.58 (2H, m), 7.13 $(2 \mathrm{H}, \mathrm{d}, J=7.6 \mathrm{~Hz}), 7.32(2 \mathrm{H}, \mathrm{d}, J=6.8 \mathrm{~Hz})$. The enantiomeric excess was determined to be $4 \%$ ee by chiral HPLC with Daicel Chiralpak AD-3 column [eluent: hexane-IPA $=4: 1$; flow rate: $1.0 \mathrm{~mL} / \mathrm{min}$; detection: $254 \mathrm{~nm} ; t_{\mathrm{R}}: 51.6 \mathrm{~min}(R), 54.3 \mathrm{~min}$ $(S)]$.

(-)-1,2-Diethyl 3,3-Dimethyl Butane-1,2,3,3-tetracarboxylate (5ea) ${ }^{46)}$ Colorless oil. $144 \mathrm{mg}$. 91\% yield. TLC: $R f$ 0.20 (hexane-EtOAc $=4: 1$, stained blue with phosphomolybdic acid). $[\alpha]_{\mathrm{D}}^{24}-22.3\left(c=1.01, \mathrm{CHCl}_{3}\right)$ for $94 \%$ ee. ${ }^{1} \mathrm{H}-\mathrm{NMR}$ $\left(\mathrm{CDCl}_{3}\right) \delta: 1.24(3 \mathrm{H}, \mathrm{t}, J=6.8 \mathrm{~Hz}), 1.26(3 \mathrm{H}, \mathrm{t}, J=7.2 \mathrm{~Hz})$, $1.47(3 \mathrm{H}, \mathrm{s}), 2.57(1 \mathrm{H}, \mathrm{dd}, J=16.4,3.2 \mathrm{~Hz}), 2.82(1 \mathrm{H}, \mathrm{dd}$,
$J=16.4,10.4 \mathrm{~Hz}), 3.69-3.72(1 \mathrm{H}, \mathrm{m}), 3.75(6 \mathrm{H}, \mathrm{s}), 4.14(2 \mathrm{H}, \mathrm{q}$, $J=6.8 \mathrm{~Hz}), 4.15(2 \mathrm{H}, \mathrm{q}, J=7.2 \mathrm{~Hz})$. The enantiomeric excess was determined to be $94 \%$ ee by chiral HPLC with Daicel Chiralpak AD-H column [eluent: hexane-IPA = 19:1; flow rate: $1.0 \mathrm{~mL} / \mathrm{min}$; detection: $220 \mathrm{~nm} ; t_{\mathrm{R}}: 13.6 \mathrm{~min}$ (major), $15.0 \mathrm{~min}$ (minor)].

(R)-3,3-Dibenzyl 1,2-Diethyl Butane-1,2,3,3-tetracarboxylate $(\mathbf{5 f a})^{46)} \quad$ Pale yellow oil. $219 \mathrm{mg}$. 93\% yield. TLC: $R f$ 0.34 (hexane-EtOAc $=4: 1$, stained white with anisaldehyde). $[\alpha]_{\mathrm{D}}^{29}-11.4\left(c=1.0, \mathrm{CHCl}_{3}\right)$ for $98 \%$ ee. ${ }^{1} \mathrm{H}-\mathrm{NMR}\left(\mathrm{CDCl}_{3}\right) \delta$ : $1.16(3 \mathrm{H}, \mathrm{t}, J=7.2 \mathrm{~Hz}), 1.24(3 \mathrm{H}, \mathrm{t}, J=7.6 \mathrm{~Hz}), 1.50(3 \mathrm{H}, \mathrm{s})$, $2.56(1 \mathrm{H}, \mathrm{dd}, J=16.6,3.2 \mathrm{~Hz}), 2.77(1 \mathrm{H}, \mathrm{dd}, J=16.6,10.4 \mathrm{~Hz})$, $3.76(1 \mathrm{H}, \mathrm{dd}, J=10.4,3.2 \mathrm{~Hz}), 4.03(2 \mathrm{H}, \mathrm{q}, J=7.2 \mathrm{~Hz}), 4.10$ $(2 \mathrm{H}, \mathrm{q}, J=7.6 \mathrm{~Hz}), 5.08(1 \mathrm{H}, \mathrm{d}, J=12.4 \mathrm{~Hz}), 5.12(2 \mathrm{H}, \mathrm{s})$, $5.14(1 \mathrm{H}, \mathrm{d}, J=12.4 \mathrm{~Hz}), 7.22-7.25(4 \mathrm{H}, \mathrm{m}), 7.30-7.32(6 \mathrm{H}$, $\mathrm{m})$. The enantiomeric excess was determined to be $98 \%$ ee by chiral HPLC with Daicel Chiralpak AD-3 column [eluent: hexane-IPA $=19: 1$; flow rate: $1.0 \mathrm{~mL} / \mathrm{min}$; detection: $254 \mathrm{~nm}$; $\left.t_{\mathrm{R}}: 29.6 \min (S), 30.8 \min (R)\right]$.

(-)-3,3-Dibenzyl 1,2-Bis(4-bromobenzyl)butane-1,2,3,3tetracarboxylate $\mathbf{( 5 f d )}^{46)}$ Pale yellow oil. $345 \mathrm{mg}$. $92 \%$ yield. TLC: $R f 0.41$ (hexane-EtOAc $=4: 1$, stained white with anisaldehyde). $[\alpha]_{\mathrm{D}}^{27}-12.8\left(c=0.69, \mathrm{CHCl}_{3}\right)$ for $93 \%$ ee. ${ }^{1} \mathrm{H}-\mathrm{NMR}\left(\mathrm{CDCl}_{3}\right) \delta$ : $1.49(3 \mathrm{H}, \mathrm{s}), 2.62(1 \mathrm{H}, \mathrm{dd}, J=16.6$, $3.2 \mathrm{~Hz}), 2.86(1 \mathrm{H}, \mathrm{dd}, J=16.6,11.0 \mathrm{~Hz}), 3.81(1 \mathrm{H}, \mathrm{dd}, J=11.0$, $3.2 \mathrm{~Hz}), 4.83-5.02(8 \mathrm{H}, \mathrm{m}), 7.08(2 \mathrm{H}, \mathrm{d}, J=8.0 \mathrm{~Hz}), 7.14-7.20$ $(6 \mathrm{H}, \mathrm{m}), 7.26-7.30(6 \mathrm{H}, \mathrm{m}), 7.41(2 \mathrm{H}, \mathrm{d}, J=8.4 \mathrm{~Hz}), 7.45(2 \mathrm{H}$, $\mathrm{d}, J=8.0 \mathrm{~Hz}$ ). The enantiomeric excess was determined to be 93\% ee by chiral HPLC with Daicel Chiralpak AD-3 column [eluent: hexane-IPA $=4: 1$; flow rate: $1.0 \mathrm{~mL} / \mathrm{min}$; detection: $254 \mathrm{~nm} ; t_{\mathrm{R}}: 31.9 \mathrm{~min}$ (minor), $40.0 \mathrm{~min}$ (major)].

(R)-3,3-Dibenzyl-1,2-diethylpentane-1,2,3,3-tetracarboxylate (5ga) ${ }^{46)}$ Colorless oil. $212 \mathrm{mg}$. $88 \%$ yield. TLC: $R f$ 0.31 (hexane-EtOAc $=4: 1$, stained white with anisaldehyde). $[\alpha]_{\mathrm{D}}^{28}+19.5\left(c=0.99, \mathrm{CHCl}_{3}\right)$ for $92 \%$ ee. ${ }^{1} \mathrm{H}-\mathrm{NMR}\left(\mathrm{CDCl}_{3}\right)$ $\delta: 0.92(3 \mathrm{H}, \mathrm{t}, J=7.6 \mathrm{~Hz}), 1.15(3 \mathrm{H}, \mathrm{t}, J=7.6 \mathrm{~Hz}), 1.24(3 \mathrm{H}, \mathrm{t}$, $J=7.2 \mathrm{~Hz}), 1.93-2.03(2 \mathrm{H}, \mathrm{m}), 2.64(1 \mathrm{H}, \mathrm{dd}, J=16.6,2.8 \mathrm{~Hz})$, $2.82(1 \mathrm{H}, \mathrm{dd}, J=16.6,10.8 \mathrm{~Hz}), 3.62(1 \mathrm{H}, \mathrm{dd}, J=10.8,2.8 \mathrm{~Hz})$, $4.01(2 \mathrm{H}, \mathrm{q}, J=7.6 \mathrm{~Hz}), 4.11(2 \mathrm{H}, \mathrm{q}, J=7.2 \mathrm{~Hz}), 5.07(2 \mathrm{H}$, $\mathrm{d}, J=12.0 \mathrm{~Hz}), 5.17(2 \mathrm{H}, \mathrm{d}, J=12.0 \mathrm{~Hz}), 7.24-7.27(2 \mathrm{H}, \mathrm{m})$, $7.29-7.32(8 \mathrm{H}, \mathrm{m})$. The ee was determined to be $92 \%$ ee by chiral HPLC with Daicel Chiralpak AS-H column [eluent: hexane-IPA $=19 / 1$; flow rate: $1.0 \mathrm{~mL} / \mathrm{min}$; detection: $254 \mathrm{~nm}$; $\left.t_{\mathrm{R}}: 7.7 \min (S), 9.6 \min (R)\right]$.

(R)-3,3-Dibenzyl 1,2-Diethyl 4-Methylpentane-1,2,3,3tetracarboxylate (5ha) $^{46)}$ Pale yellow oil. 226 mg. $91 \%$ yield. TLC: $R f 0.37$ (hexane-EtOAc $=4: 1$, stained blue with phosphomolybdic acid). $[\alpha]_{\mathrm{D}}^{26}+23.7\left(c=0.7, \mathrm{CHCl}_{3}\right)$ for $95 \%$ ee. ${ }^{1} \mathrm{H}-\mathrm{NMR}\left(\mathrm{CDCl}_{3}\right) \delta: 1.01(3 \mathrm{H}, \mathrm{d}, J=6.4 \mathrm{~Hz}), 1.02(3 \mathrm{H}, \mathrm{d}$, $J=6.4 \mathrm{~Hz}), 1.09(3 \mathrm{H}, \mathrm{t}, J=6.8 \mathrm{~Hz}), 1.24(3 \mathrm{H}, \mathrm{t}, J=6.8 \mathrm{~Hz})$, 2.43-2.51 (2H, m), $2.67(1 \mathrm{H}, \mathrm{dd}, J=16.4,11.6 \mathrm{~Hz}), 3.75(1 \mathrm{H}$, $\mathrm{dd}, J=11.6,2.0 \mathrm{~Hz}), 3.91(2 \mathrm{H}, \mathrm{q}, J=6.8 \mathrm{~Hz}), 4.12(2 \mathrm{H}, \mathrm{q}$, $J=6.8 \mathrm{~Hz}), 5.07(1 \mathrm{H}, \mathrm{d}, J=12.4 \mathrm{~Hz}), 5.13(1 \mathrm{H}, \mathrm{d}, J=12.4 \mathrm{~Hz})$, $5.19(2 \mathrm{H}, \mathrm{d}, J=12.4 \mathrm{~Hz}), 7.29-7.32(10 \mathrm{H}, \mathrm{m})$. The ee was determined to be $95 \%$ ee by chiral HPLC with Daicel Chiralpak AD-3 column [eluent: hexane-IPA $=19: 1$; flow rate: $1.0 \mathrm{~mL} / \mathrm{min}$; detection: $\left.254 \mathrm{~nm} ; t_{\mathrm{R}}: 20.1 \mathrm{~min}(R), 22.8 \mathrm{~min}(S)\right]$.

(+)-1,2-Dibenzyl 3,3-Diethyl 4-Methylpentane-1,2,3,3tetracarboxylate $\left(\mathbf{5 i c}^{46)} \quad\right.$ Pale yellow oil. $196 \mathrm{mg} . \quad 79 \%$ yield. TLC: $R f 0.37$ (hexane-EtOAc $=4: 1$, stained blue with phosphomolybdic acid). $[\alpha]_{435}^{24}+11.5\left(c=0.7, \mathrm{CHCl}_{3}\right)$ for $90 \%$ 
ee. ${ }^{1} \mathrm{H}-\mathrm{NMR}\left(\mathrm{CDCl}_{3}\right) \delta$ : $1.01(3 \mathrm{H}, \mathrm{d}, J=7.6 \mathrm{~Hz}), 1.03(3 \mathrm{H}, \mathrm{d}$, $J=7.6 \mathrm{~Hz}), 1.16(3 \mathrm{H}, \mathrm{t}, J=7.6 \mathrm{~Hz}), 1.22(3 \mathrm{H}, \mathrm{t}, J=7.2 \mathrm{~Hz})$, $2.38(1 \mathrm{H}$, sept, $J=7.6 \mathrm{~Hz}), 2.58(1 \mathrm{H}, \mathrm{dd}, J=17.0,2.0 \mathrm{~Hz})$, $2.80(1 \mathrm{H}, \mathrm{dd}, J=17.0,11.2 \mathrm{~Hz}), 3.82(1 \mathrm{H}, \mathrm{dd}, J=11.2,2.0 \mathrm{~Hz})$, 3.95-4.17 (4H, m), 5.01-5.13 (4H, m), 7.26-7.32 (10H, m). The ee was determined to be $90 \%$ ee by chiral HPLC with Daicel Chiralcel OD-H column [eluent: hexane-IPA $=19: 1$; flow rate: $1.0 \mathrm{~mL} / \mathrm{min}$; detection: $254 \mathrm{~nm} ; t_{\mathrm{R}}: 10.7 \mathrm{~min}$ (minor), $13.4 \min$ (major)].

(+)-3,3-Dibenzyl 1,2-Diethyl 4-Phenylbutane-1,2,3,3tetracarboxylate (5ja) Pale yellow oil. $98 \mathrm{mg}$. 36\% yield. TLC: $R f 0.39$ (hexane-EtOAc $=4: 1$, stained blue with phosphomolybdic acid). $[\alpha]_{435}^{26}+5.6(c=1.3, \mathrm{EtOH})$ for $86 \%$ ee. IR (ATR) $\mathrm{cm}^{-1}: 3065,3033,2981,1727,1173 .{ }^{1} \mathrm{H}-\mathrm{NMR}\left(\mathrm{CDCl}_{3}\right)$ $\delta: 1.20(3 \mathrm{H}, \mathrm{t}, J=7.6 \mathrm{~Hz}), 1.21(3 \mathrm{H}, \mathrm{t}, J=7.2 \mathrm{~Hz}), 2.75(1 \mathrm{H}$, $\mathrm{dd}, J=16.8,2.8 \mathrm{~Hz}), 2.88(1 \mathrm{H}, \mathrm{dd}, J=16.8,10.8 \mathrm{~Hz}), 3.33$ $(2 \mathrm{H}, \mathrm{s}), 3.65(1 \mathrm{H}, \mathrm{dd}, J=10.8,2.8 \mathrm{~Hz}), 4.06-4.12(4 \mathrm{H}, \mathrm{m})$, $4.96(2 \mathrm{H}, \mathrm{s}), 5.09(2 \mathrm{H}, \mathrm{s}), 7.12-7.14(2 \mathrm{H}, \mathrm{m}), 7.21-7.31(13 \mathrm{H}$, m). ${ }^{13} \mathrm{C}-\mathrm{NMR}\left(\mathrm{CDCl}_{3}\right) \quad \delta$ : 13.7, 14.1, 33.8, 39.6, 45.4, 60.6, $60.8,61.3,67.4,67.5,127.2,128.2,128.3,128.3,128.4,128.4$, $128.4,128.4,130.5,134.6,134.9,135.4,168.7,169.0,171.8$ (one carbon overlapped). FAB-MS m/z: 547.2333 (Calcd for $\mathrm{C}_{32} \mathrm{H}_{35} \mathrm{O}_{8}:$ 547.2332). MS $m / z: 547\left(\mathrm{M}+\mathrm{H}^{+}\right), 455$, 91. The ee was determined to be $86 \%$ ee by chiral HPLC with Daicel Chiralpak AD-3 column [eluent: hexane-IPA = 19:1; flow rate: $1.0 \mathrm{~mL} / \mathrm{min}$; detection: $254 \mathrm{~nm} ; t_{\mathrm{R}}: 33.7 \mathrm{~min}$ (major), $38.7 \mathrm{~min}$ (minor)].

(-)-1,2-Dibenzyl 3,3-Diethyl 4-Phenylbutane-1,2,3,3tetracarboxylate (5kc) Colorless oil. $120 \mathrm{mg} .44 \%$ yield. TLC: Rf 0.42 (hexane-EtOAc $=4: 1$, stained blue with phosphomolybdic acid). $[\alpha]_{435}^{24}-5.9(c=1.3, \mathrm{MeOH})$ for $79 \%$ ee. IR (ATR) $\mathrm{cm}^{-1}: 3033,1728,1155 .{ }^{1} \mathrm{H}-\mathrm{NMR}\left(\mathrm{CDCl}_{3}\right) \delta: 1.09(3 \mathrm{H}$, t, $J=6.8 \mathrm{~Hz}), 1.14(3 \mathrm{H}, \mathrm{t}, J=7.2 \mathrm{~Hz}), 2.84(1 \mathrm{H}, \mathrm{dd}, J=16.8$, $3.2 \mathrm{~Hz}), 2.97(1 \mathrm{H}, \mathrm{dd}, J=16.8,11.2 \mathrm{~Hz}), 3.28(2 \mathrm{H}, \mathrm{s}) 3.71(1 \mathrm{H}$, $\mathrm{dd}, J=11.2,3.2 \mathrm{~Hz}), 3.95-4.08(4 \mathrm{H}, \mathrm{m}), 4.99-5.15(4 \mathrm{H}, \mathrm{m})$, 7.18-7.22 (5H, m), 7.27-7.37 (10H, m). ${ }^{13} \mathrm{C}-\mathrm{NMR}\left(\mathrm{CDCl}_{3}\right) \delta$ : 13.7, 13.8, 25.3, 33.9, 39.5, 45.3, 60.6, 61.6, 66.5, 67.3, 127.0, $128.0,128.2,128.3,128.4,128.5,128.6,130.5,135.2,135.6$, 135.7, 169.0, 169.1, 171.6 (two carbons overlapped). FABMS $m / z$ : 547.2335 (Calcd for $\mathrm{C}_{32} \mathrm{H}_{35} \mathrm{O}_{8}$ : 547.2332). MS $m / z$ : $547\left(\mathrm{M}+\mathrm{H}^{+}\right), 455,91$. The ee was determined to be $79 \%$ ee by chiral HPLC with Daicel Chiralpak AD-H column [eluent: hexane-IPA $=9: 1$; flow rate: $1.0 \mathrm{~mL} / \mathrm{min}$; detection: $254 \mathrm{~nm}$; $t_{\mathrm{R}}: 17.2 \mathrm{~min}$ (minor), $18.9 \mathrm{~min}$ (major)].

(-)-Tetraethyl 1-Chloropropane-1,1,2,3-tetracarboxylate (5la) Pale yellow oil. $144 \mathrm{mg}$. 79\% yield. TLC: $R f 0.53$ (hexane-EtOAc $=4: 1$, stained yellow with phosphomolybdic acid). $[\alpha]_{435}^{27}-8.1\left(c=1.07, \mathrm{CHCl}_{3}\right)$ for $74 \%$ ee. IR (ATR) $\mathrm{cm}^{-1}: 2984,1732,1239,1199,1175,1023 .{ }^{1} \mathrm{H}-\mathrm{NMR}\left(\mathrm{CDCl}_{3}\right) \delta$ : $1.21-1.33(12 \mathrm{H}, \mathrm{m}), 2.83(1 \mathrm{H}, \mathrm{dd}, J=16.9,3.2 \mathrm{~Hz}), 2.94(1 \mathrm{H}$, dd, $J=16.9,9.6 \mathrm{~Hz}), 4.09-4.11(1 \mathrm{H}, \mathrm{m}), 4.14-4.21(4 \mathrm{H}, \mathrm{m})$, 4.28-4.32 (4H, m). ${ }^{13} \mathrm{C}-\mathrm{NMR}\left(\mathrm{CDCl}_{3}\right) \delta: 13.7,13.8,14.1,29.7$, 33.0, 47.9, 61.0, 61.8, 63.4, 63.5, 71.1, 165.1, 165.6, 169.3, 171.3. FAB-MS $m / z$ : 367.1181 (Calcd for $\mathrm{C}_{15} \mathrm{H}_{24} \mathrm{O}_{8} \mathrm{Cl}$ : 367.1160). MS $m / z: 367\left(\mathrm{M}+\mathrm{H}^{+}\right)$. The ee was determined to be $74 \%$ ee by chiral HPLC with Daicel Chiralpak AD-3 column [eluent: hexane-IPA $=9: 1$; flow rate: $1.0 \mathrm{~mL} / \mathrm{min}$; detection: $220 \mathrm{~nm}$; $t_{\mathrm{R}}: 12.0 \mathrm{~min}$ (major), $13.9 \mathrm{~min}$ (minor)].

(S)-1,1-Dibenzyl 2,3-Diethyl 1-Fluoropropane-1,1,2,3tetracarboxylate (5ma) Pale yellow oil. $215 \mathrm{mg}$. $91 \%$ yield. TLC: $R f 0.53$ (hexane-EtOAc $=4: 1$, stained blue with phos- phomolybdic acid). $[\alpha]_{\mathrm{D}}^{29}-9.1\left(c=1.0, \mathrm{CHCl}_{3}\right)$ for $91 \%$ ee. IR (ATR) $\mathrm{cm}^{-1}: 3067,1734,1215,1024 .{ }^{1} \mathrm{H}-\mathrm{NMR}\left(\mathrm{CDCl}_{3}\right) \delta$ : $1.14(3 \mathrm{H}, \mathrm{t}, J=7.3 \mathrm{~Hz}), 1.24(3 \mathrm{H}, \mathrm{t}, J=6.9 \mathrm{~Hz}), 2.47(1 \mathrm{H}, \mathrm{dd}$, $J=16.9,3.7 \mathrm{~Hz}), 2.83(1 \mathrm{H}, \mathrm{dd}, J=16.9,10.1 \mathrm{~Hz}), 4.02-4.17$ $(5 \mathrm{H}, \mathrm{m}), 5.21(4 \mathrm{H}, \mathrm{s}), 7.28-7.37(10 \mathrm{H}, \mathrm{m}) .{ }^{13} \mathrm{C}-\mathrm{NMR}\left(\mathrm{CDCl}_{3}\right) \delta$ : 13.7, 14.0, 31.1, 31.1, 45.8 (d), 61.5 (d), 68.4 (d), 68.6 (d), 94.3 (d) $128.3,128.4,128.6,128.6,128.7,134.1,134.3,134.4,164.2$ (d), 164.6 (d), 168.7 (d), 170.6. FAB-MS $m / z$ : 475.1738 (Calcd for $\mathrm{C}_{25} \mathrm{H}_{28} \mathrm{O}_{8} \mathrm{~F}$ : 475.1768). MS $m / z$ : $475\left(\mathrm{M}+\mathrm{H}^{+}\right)$, 91. The ee was determined to be $91 \%$ ee by chiral HPLC with Daicel Chiralpak AD-3 column [eluent: hexane-IPA = 9: 1; flow rate: $1.0 \mathrm{~mL} / \mathrm{min}$; detection: $\left.254 \mathrm{~nm} ; t_{\mathrm{R}}: 22.9 \mathrm{~min}(S), 27.8 \mathrm{~min}(R)\right]$.

(-)-Tetraethyl 1-Fluoropropane-1,1,2,3-tetracarboxylate (5na) Pale yellow oil. $129 \mathrm{mg}$. 74\% yield. TLC: $R f 0.26$ (hexane-EtOAc $=4: 1$, stained white with anisaldehyde). $[\alpha]_{\mathrm{D}}^{29}-10.3\left(c=0.88, \mathrm{CHCl}_{3}\right)$ for $98 \%$ ee. IR (ATR) $\mathrm{cm}^{-1}$ : 2985, 1735, 1231. ${ }^{1} \mathrm{H}-\mathrm{NMR}\left(\mathrm{CDCl}_{3}\right) \delta: 1.24(3 \mathrm{H}, \mathrm{t}, J=7.3 \mathrm{~Hz})$, $1.27(3 \mathrm{H}, \mathrm{t}, J=7.3 \mathrm{~Hz}), 1.33(3 \mathrm{H}, \mathrm{t}, J=7.3 \mathrm{~Hz}), 1.33(3 \mathrm{H}$, t, $J=7.3 \mathrm{~Hz}), 2.57(1 \mathrm{H}, \mathrm{dd}, J=16.5,3.7 \mathrm{~Hz}), 2.88(1 \mathrm{H}, \mathrm{dd}$, $J=16.5,10.1 \mathrm{~Hz}), 4.04 \quad(1 \mathrm{H}, \quad \mathrm{ddd}, J=26.8,10.1,3.7 \mathrm{~Hz})$, 4.14-4.20 (4H, m), 4.29-4.35 (4H, m). ${ }^{13} \mathrm{C}-\mathrm{NMR}\left(\mathrm{CDCl}_{3}\right) \delta$ : 13.7, 14.0, 29.5, 31.1, 45.6 (d), 61.4 (d), 63.0 (d), 93.0 (d), 164.4 (d), 164.7 (d), 168.7, 170.7 (three carbons overlapped). FABMS $m / z$ : 351.1463 (Calcd for $\mathrm{C}_{15} \mathrm{H}_{24} \mathrm{O}_{8} \mathrm{~F}: 351.1455$ ). MS $m / z$ : $351\left(\mathrm{M}+\mathrm{H}^{+}\right)$. The ee was determined to be $98 \%$ ee by chiral HPLC with Daicel Chiralpak AD-H column [eluent: hexane-IPA $=19: 1$; flow rate: $1.0 \mathrm{~mL} / \mathrm{min}$; detection: $220 \mathrm{~nm} ; t_{\mathrm{R}}$ : $14.5 \mathrm{~min}$ (major), $15.5 \mathrm{~min}$ (minor)].

Typical Procedure of Transesterification (Chart 1) Under argon atmosphere, $\mathrm{Ti}(\mathrm{OEt})_{4}(0.21 \mathrm{~mL}, 1.0 \mathrm{mmol}, 4.0 \mathrm{eq})$ in EtOH $(2 \mathrm{~mL})$ was added Michael adduct 3bb $(69.0 \mathrm{mg}$, $0.25 \mathrm{mmol})$ in EtOH $(1 \mathrm{~mL})$. After refluxing for $14 \mathrm{~h}$, the reaction was quenched with sat. $\mathrm{NH}_{4} \mathrm{Cl}$ aq. $(2 \mathrm{~mL})$ and stirred for $0.5 \mathrm{~h}$. The aqueous layer was extracted with EtOAc $(3 \times 10 \mathrm{~mL})$. The combined organic layers were washed with brine $(20 \mathrm{~mL})$, and dried over $\mathrm{Na}_{2} \mathrm{SO}_{4}$. After filtration and concentration, the crude product was purified by column chromatography (hexane-EtOAc $=9: 1, \mathrm{SiO}_{2}: 5 \mathrm{~g}$ ) to give product 3ca as a pale yellow oil $(76 \mathrm{mg}, 91 \%$ yield, $81 \%$ ee).

Typical Procedure of Alkylation of Michael Adduct 3aa (Chart 2) Under argon atmosphere, methyl iodide $(0.024 \mathrm{~mL}, 0.4 \mathrm{mmol}, 4.0 \mathrm{eq})$ was added to a solution of $\mathrm{Cs}_{2} \mathrm{CO}_{3}(45.6 \mathrm{mg}, 0.14 \mathrm{mmol}, 1.4 \mathrm{eq})$ and Michael adduct 3aa ( $45.6 \mathrm{mg}, 0.1 \mathrm{mmol}, 1.0 \mathrm{eq})$ in DMF $(1 \mathrm{~mL})$ at r.t. After $14 \mathrm{~h}$, the reaction was quenched with water $(2 \mathrm{~mL})$ and stirred for $0.5 \mathrm{~h}$. The aqueous layer was extracted with EtOAc $(3 \times 10 \mathrm{~mL})$. The combined organic layers were washed with brine $(20 \mathrm{~mL})$, and dried over $\mathrm{Na}_{2} \mathrm{SO}_{4}$. After filtration and concentration, the crude product was purified by column chromatography (hexane-EtOAc $=9: 1, \mathrm{SiO}_{2}: 5 \mathrm{~g}$ ) to give product $\mathbf{5 f a}$ as a pale yellow oil $(24 \mathrm{mg}, 50 \%$ yield, $89 \%$ ee).

Procedure of Fluorination of Michael Adduct 3aa (Chart 2) Under argon atmosphere, solution of Michael adduct 3aa $(68.4 \mathrm{mg}, 0.15 \mathrm{mmol}, 1.0 \mathrm{eq})$ in DMF $(3 \mathrm{~mL})$ was added $\mathrm{NaH}(7.2 \mathrm{mg}, 0.18 \mathrm{mmol}, 1.2 \mathrm{eq}$ content; $60 \%$ by weight in oil) at $0^{\circ} \mathrm{C}$ and stirred for $0.5 \mathrm{~h}$. Then Selectfluor ${ }^{\circledR}(69.1 \mathrm{mg}$, $0.20 \mathrm{mmol}, 1.3 \mathrm{eq}$ ) was added to the reaction mixture and stirred $14 \mathrm{~h}$ at r.t. The reaction solution was poured into ice water $(5 \mathrm{~mL})$. The aqueous layer was extracted with $\mathrm{Et}_{2} \mathrm{O}$ $(3 \times 10 \mathrm{~mL})$. The combined organic layers were washed with brine $(20 \mathrm{~mL})$, and dried over $\mathrm{Na}_{2} \mathrm{SO}_{4}$. After filtration and 
concentration, the crude product was purified by column chromatography (hexane-EtOAc $=9: 1, \mathrm{SiO}_{2}: 5 \mathrm{~g}$ ) to give product 5ma as a yellow oil (64 mg, 90\% yield, 88\% ee).

Procedure of Krapcho Reaction (Chart 3) Michael adduct 3ag $(160 \mathrm{mg}, 0.3 \mathrm{mmol})$ and $\mathrm{H}_{2} \mathrm{O}(0.16 \mathrm{~mL}, 9.0 \mathrm{mmol}$, $30 \mathrm{eq}$ were stirred at $150^{\circ} \mathrm{C}$. After $40 \mathrm{~h}$, the crude product was loaded directly onto column chromatography and purified (hexane-EtOAc $=12: 1, \mathrm{SiO}_{2}: 10 \mathrm{~g}$ ) to give product $\mathbf{6 a g}$ as a colorless oil ( $78.9 \mathrm{mg}, 76 \%$ yield, $90 \%$ ee).

(-)-1,2-Diallyl 3-Benzyl-propane-1,2,3-tricarboxylate (6ag) Colorless oil. $78.9 \mathrm{mg}$. $76 \%$ yield. TLC: Rf 0.47 (hexane-EtOAc $=4: 1$, stained blue with phosphomolybdic acid). $[\alpha]_{435}^{20}-5.2\left(c=1.05, \mathrm{CHCl}_{3}\right)$ for $90 \%$ ee. IR (ATR) $\mathrm{cm}^{-1}: 3034,1729,1649,1152 .{ }^{1} \mathrm{H}-\mathrm{NMR}\left(\mathrm{CDCl}_{3}\right) \delta: 2.62-2.71$ (2H, m), 2.79-2.88 (2H, m), 3.30-3.35 (1H, m), 4.57 (4H, t, $J=5.5 \mathrm{~Hz}), 5.13(2 \mathrm{H}, \mathrm{s}), 5.20-5.33(4 \mathrm{H}, \mathrm{m}), 5.80-5.93(2 \mathrm{H}, \mathrm{m})$, 7.33-7.39 (5H, m). ${ }^{13} \mathrm{C}-\mathrm{NMR}\left(\mathrm{CDCl}_{3}\right) \delta: 35.2,35.2,37.4,65.5$, 65.7, 66.6, 118.4, 118.5, 128.3, 128.3, 128.5, 131.8, 131.8, 135.6, 170.9, 171.1, 172.7. FAB-MS $m / z: 347.1533$ (Calcd for $\mathrm{C}_{19} \mathrm{H}_{23} \mathrm{O}_{6}$ : 347.1495). MS m/z: $347\left(\mathrm{M}+\mathrm{H}^{+}\right)$. The ee was determined to be $90 \%$ ee by chiral HPLC with Daicel Chiralpak AD-3 column [eluent: hexane-IPA $=19: 1$; flow rate: $1.0 \mathrm{~mL} / \mathrm{min}$; detection: $254 \mathrm{~nm}$; $t_{\mathrm{R}}$ : $18.5 \mathrm{~min}$ (major), $19.7 \mathrm{~min}$ (minor)].

Acknowledgments This work was partially supported by Naito Foundation and the Ministry of Education, Science and Culture of Japan.

Conflict of Interest The authors declare no conflict of interest.

\section{References and Notes}

1) Christoffers J., Koripelly G., Rosiak A., Rössle M., Synthesis, 2007, 1279-1300 (2007).

2) Tsogoeva S. B., Eur. J. Org. Chem., 2007, 1701-1716 (2007).

3) Almaşi D., Alonso D. A., Nájera C., Tetrahedron Asymmetry, 18 299-365 (2007).

4) Zhang Y., Wang W., Catal. Sci. Technol., 2, 42-53 (2012).

5) Matsunaga S., "Comprehensive Chirality," Vol. 5, ed. by Carreira E. M., Yamamoto H., Elsevier, Amsterdam, 2012, pp. 243-292.

6) Takemoto Y., Stadler M., "Comprehensive Chirality," Vol. 6, ed. by Carreira E. M., Yamamoto H., Elsevier, Amsterdam, 2012, pp. 37-68.

7) Zheng K., Liu X., Feng X., Chem. Rev., 118, 7586-7656 (2018).

8) Evans D. A., Rovis T., Kozlowski M. C., Downey C. W., Tedrow J. S., J. Am. Chem. Soc., 122, 9134-9142 (2000).

9) Ooi T., Ohara D., Fukumoto K., Maruoka K., Org. Lett., 7, $3195-$ $3197(2005)$.

10) Wang J., Li H., Zu L., Jiang W., Xie H., Duan W., Wang W., J. Am. Chem. Soc., 128, 12652-12653 (2006).

11) Naka H., Kanase N., Ueno M., Kondo Y., Chem. Eur. J., 14, $5267-$ 5274 (2008).

12) Agostinho M., Kobayashi S., J. Am. Chem. Soc., 130, 2430-2431 (2008).

13) Wascholowski V., Knudsen K. R., Mitchell C. E. T., Ley S. V., Chem. Eur. J., 14, 6155-6165 (2008).

14) Chen D., Chen Z., Xiao X., Yang Z., Lin L., Liu X., Feng X., Chem. Eur. J., 15, 6807-6810 (2009).

15) Mase N., Fukasawa M., Kitagawa N., Shibagaki F., Noshiro N., Takabe K., Synlett, 2010, 2340-2344 (2010).

16) Mao Z., Jia Y., Li W., Wang R., J. Org. Chem., 75, 7428-7430 (2010).

17) Yoshida M., Narita M., Hara S., J. Org. Chem., 76, 8513-8517
(2011).

18) Espinosa M., Blay G., Cardona L., Pedro J. R., Chem. Eur. J., 19, 14861-14866 (2013).

19) Tsubo T., Yamada T., Synlett, 26, 1111-1115 (2015).

20) Lippur K., Kaabel S., Järving I., Rissanen K., Kanger T., J. Org. Chem., 80, 6336-6341 (2015).

21) Ye W., Jiang Z., Zhao Y., Goh S. L. M., Leow D., Soh Y.-T., Tan C.-H., Adv. Synth. Catal., 349, 2454-2458 (2007).

22) Gómez-Torres E., Alonso D. A., Gómez-Bengoa E., Nájera C., Org. Lett., 13, 6106-6109 (2011).

23) Clarke H. T., Murray T. F. Org. Synth., Coll., I, 272-273 (1941).

24) Tanaka K., Kukita K., Ichibakase T., Kotani S., Nakajima M., Chem. Commun., 47, 5614-5616 (2011).

25) Ichibakase T., Nakatsu M., Nakajima M., Molecules, 16, 5008-5019 (2011).

26) Ichibakase T., Nakajima M., Org. Lett., 13, 1579-1581 (2011).

27) Ichibakase T., Nakajima M., Synthesis, 44, 3145-3151 (2012).

28) Ichibakase T., Kaneko T., Orito Y., Kotani S., Nakajima M., Tetrahedron, 68, 4210-4224 (2012).

29) Kotani S., Kukita K., Tanaka K., Ichibakase T., Nakajima M., J. Org. Chem., 79, 4817-4825 (2014).

30) Kotani S., Moritani M., Nakajima M. Asian, J. Org. Chem., 4, 616-618 (2015).

31) Osakama K., Nakajima M., Org. Lett., 18, 236-239 (2016).

32) Kotani S., Asano T., Moritani M., Nakajima M., Tetrahedron Lett., 57, 4217-4219 (2016)

33) Nakajima M., Watanabe R., Osakama K., Sakamoto M., Takemoto D., Kukita K., Heterocycles, in press.10.3987/COM-18-S(T)63

34) Selected reports for the catalytic asymmetric Michael reactions using alkali metal salts of binaphthol derivatives: Tamai Y., Kamifuku A., Koshiishi E., Miyano S., Chem. Lett., 24, 957-958 (1995).

35) Kim Y. S., Matsunaga S., Das J., Sekine A., Ohshima T., Shibasaki M., J. Am. Chem. Soc., 122, 6506-6507 (2000).

36) Belokon Y. N., Gugkaeva Z. T., Maleev V. I., Moskalenko M. A., Tsaloev A. T., Khrustalev V. N., Hakobyan K. V., Tetrahedron Asymmetry, 22, 167-172 (2011).

37) Otani T., Sugawara A., Tamai Y., Tetrahedron Lett., 55, 4923-4926 (2014).

38) Samoilichenko Y., Kondratenko V., Ezernitskaya M., Lyssenko K., Peregudov A., Khrustalev V., Maleev V., Moskalenko M., North M., Tsaloev A., Gugkaeva Z. T., Belokon Y., Catal. Sci. Technol., 7, 90-101 (2017).

39) Selected examples of lithium binaphtholate-catalyzed asymmetric reactions: Schiffers R., Kagan H. B., Synlett, 1175-1178 (1997).

40) Loog O., Mäeorg U., Tetrahedron Asymmetry, 10, 2411-2415 (1999). 41) Holmes I. P., Kagan H. B., Tetrahedron Lett., 41, 7453-7456 (2000).

42) Holmes I. P., Kagan H. B., Tetrahedron Lett., 41, 7457-7460 (2000).

43) Hatano M., Ikeno T., Miyamoto T., Ishihara K., J. Am. Chem. Soc., 127, 10776-10777 (2005).

44) Hatano M., Ishihara K., Synthesis, 3785-3801 (2010).

45) Hatano M., Horibe T., Ishihara K., J. Am. Chem. Soc., 132, 56-57 (2010)

46) Sakamoto M., Kaneko T., Orito Y., Nakajima M., Synlett, 27, $2477-$ 2480 (2016).

47) Hanessian S., Gomtsyan A., Payne A., Herve Y., Beaudoin S., J. Org. Chem., 58, 5032-5034 (1993).

48) Harmat N. J. S., Mangani S., Perrotta E., Giannotti D., Nannicini R., Altamura M., Tetrahedron Lett., 41, 1261-1264 (2000).

49) Lithium salt prepared from the $(R)-3,3^{\prime}-\mathrm{Cl}_{2}-\mathrm{BINOL}(10 \mathrm{~mol} \%)$ and lithium hydroxide $(20 \mathrm{~mol} \%$ ) gave lesser result ( $68 \%$ yield, $75 \%$ ee). Mono-lithium salt prepared from $(R)-3,3^{\prime}-\mathrm{Cl}_{2}$-BINOL (10 mol\%) and ${ }^{n} \mathrm{BuLi}(10 \mathrm{~mol} \%$ ) afforded lesser result ( $76 \%$ yield, $74 \%$ ee).

50) Žari S., Kailas T., Kudrjashova M., Öeren M., Järving I., Tamm T., Lopp M., Kanger T., Beilstein J. Org. Chem., 8, 1452-1457 (2012).

51) Hale J., Lynch C., Caldwell C., Willoughby C., Kim D., Shen D., Mills S., Chapman K., Chen L., Gentry A., MacCoss M., Konteatis 
Z., US20020094989.

52) Li H., Gao X., Liu R., Wang Y., Zhang M., Fu Z., Mi Y., Wang Y., Yao Z., Gao Q., Eur. J. Med. Chem., 101, 400-408 (2015).

53) Fumonisins are common contaminant of corn: Bezuidenhout S. C., Gelderblom W. C. A., Gorst-Allman C. P., Horak R. M., Marasas W. F. O., Spiteller G., Vleggaar R., J. Chem. Soc., Chem. Commun., $743-745$ (1988)

54) Gelderblom W. C. A., Jaskiewicz K., Marasas W. F. O., Thiel P. G., Horak R. M., Vleggaar R., Kriek N. P., Appl. Environ. Microbiol., 54, 1806-1811 (1988).

55) Rheeder J. P., Marasas W. F. O., Thiel P. G., Sydenham E. W., Shephard G. S., van Schalkwyk D. J., Phytopathology, 82, 353-357 (1992).

56) Hoye T. R., Jiménez J. I., Shier W. T., J. Am. Chem. Soc., 116, 9409-9410 (1994)

57) Boyle C. D., Kishi Y., Tetrahedron Lett., 36, 4579-4582 (1995).

58) Shi Y., Peng L. F., Kishi Y., J. Org. Chem., 62, 5666-5667 (1997).

59) Humpf H.-U., Schmelz E.-M., Meredith F. I., Vesper H., Vales T. R. Wang E., Menaldino D. S., Liotta D. C., Merrill A. H. Jr., J. Biol. Chem., 273, 19060-19064 (1998).

60) Sadler T. W., Merrill A. H., Stevens V. L., Sullards M. C., Wang E., Wang P., Teratology, 66, 169-176 (2002).

61) Hendricks K., Epidemiology, 10, 198-200 (1999).

62) Pereira C. L., Chen Y.-H., McDonald F. E., J. Am. Chem. Soc., 131, 6066-6067 (2009).

63) McDonald F. E., Pereira C. L., Chen Y.-H., Pure Appl. Chem., 83, 445-459 (2011)

64) J-104 118 inhibits squalene synthase: Iwasawa Y., Hayashi M., Nomoto T., Shibata J., Mitsuya M., Hirota K., Yonemoto M., Kamei T., Miura K., Tomimoto K., Bioorg. Med. Chem. Lett., 5, 1989-1994
(1995).

65) Iwasawa Y., Shibata J., Nonoshita K., Arai S., Masaki H., Tomimoto K., Tetrahedron, 52, 13881-13894 (1996).

66) Iwasawa Y., Nonoshita K., Tomimoto K., Tetrahedron Lett., 36, 7459-7462 (1995)

67) Iwasawa Y., Shibata J., Mitsuya M., Masaki H., Hayashi M., Kanno T., Sawasaki Y., Hisaka A., Kamei T., Tomimoto K., Bioorg. Med. Chem. Lett., 6, 463-466 (1996).

68) The procedure of acidic condition: Under $\mathrm{H}_{2}$ atmosphere, Pd-C ( $31.9 \mathrm{mg}, 0.03 \mathrm{mmol}, 10 \%$ of the weight of the substrate) in EtOH $(1.0 \mathrm{~mL})$ was added Michael product 3aa $(160 \mathrm{mg}, 0.3 \mathrm{mmol})$ in EtOH $(3.0 \mathrm{~mL})$. After stirring for $0.5 \mathrm{~h}$, the reaction mixture was filtered using a celite cake and the filtrate was concentrated in vacuo. The crude product 2-(1,4-diethoxy-1,4-dioxobutan-2-yl)malonic acid was used without further purification. A solution of obtained dicarboxylic acid in $\mathrm{HCl}(5.0 \mathrm{~mL}, 35 \%$ in weight) was refluxed for $5 \mathrm{~h}$

69) The procedure of standard Krapcho reaction: Michael adduct 3 ag $(160 \mathrm{mg}, 0.3 \mathrm{mmol}), \mathrm{H}_{2} \mathrm{O}(0.05 \mathrm{~mL}$. $3.0 \mathrm{mmol}, 10 \mathrm{eq}$ and $\mathrm{LiCl}$ $\left(0.6 \mathrm{mmol}, 2.0 \mathrm{eq}\right.$ were stirred at $150^{\circ} \mathrm{C}$ in DMSO. After $11 \mathrm{~h}$, the crude product was loaded directly onto column chromatography and purified (hexane-EtOAc $=12: 1, \mathrm{SiO}_{2}: 10 \mathrm{~g}$ ) to give product $6 \mathbf{a g}$ as a colorless oil $(27.0 \mathrm{mg}, 26 \%$ yield, $87 \%$ ee).

70) Krapcho A. P., Synthesis, 1982, 805-822 (1982).

71) Krapcho A. P., Synthesis, 1982, 893-914 (1982).

72) Poon P. S., Banerjee A. K., Laya M. S., J. Chem. Res., 35, 67-73 (2011).

73) Krapcho A. P., ARKIVOC, 1-53 (2007).

74) Evans D. A., Scheidt K. A., Downey C. W., Org. Lett., 3, 3009-3012 (2001). 